\title{
L'Homme
}

LHOMME Revue française d'anthropologie

167-168 | 2003

Passages à l'âge d'homme

\section{Aux files des âges}

Classes d'âge et générations dans cinq régions dogon (Mali)

Éric Jolly

\section{(2) OpenEdition}

Journals

Édition électronique

URL : http://journals.openedition.org/lhomme/21497

DOI : 10.4000//homme.21497

ISSN : 1953-8103

Éditeur

Éditions de l'EHESS

\section{Édition imprimée}

Date de publication : 1 décembre 2003

Pagination : 67-104

ISSN : 0439-4216

Référence électronique

Éric Jolly, "Aux files des âges », L'Homme [En ligne], 167-168 | 2003, mis en ligne le 01 janvier 2005 consulté le 02 mai 2019. URL : http://journals.openedition.org//homme/21497 ; DOI : 10.4000/ Ihomme.21497 


\section{Aux files des âges \\ Classes d'âge et générations dans cinq régions dogon (Mali)}

Éric Jolly

À la mémoire de Marcel Kervran

LE THÈME des classes d'âge n'a guère inspiré les générations de chercheurs qui se sont succédé en pays dogon depuis plus de soixante ans. Seuls deux anthropologues ont ébauché une telle étude dans leur monographie respective. Denise Paulme a consacré un chapitre de sa thèse aux "fraternités d'âge" dogon, en insistant sur la solidarité entre membres d'une même promotion de circoncis (1940 : 236-247). Quant à Jacky Bouju, il s'est intéressé au contraire à la "stratification " de la population adulte en reconstituant le "système d'échelons d'âge " du village de SibiSibi (1984: 67-77) et en glissant quelques indices sur sa dimension générationnelle (ibid. : 73,75 ).

À partir des pistes ouvertes par Denise Paulme et Jacky Bouju, cet article réexamine l'organisation d'âge dogon à travers cinq études de cas, choisis dans des régions voisines aux structures politiques assez contrastées (en zones linguistiques t $\varepsilon$ ne, dono, tom $\supset$ et torว). Certains douteront peutêtre de l'intérêt d'une telle démarche appliquée à une société où les classes d'âge sont peu saillantes, comparées aux modèles habituellement décrits. Or, se détacher provisoirement des systèmes d'âge classiques pour se plonger dans la complexité des matériaux dogon fait justement apparaître une variété inattendue de formules, souvent inédites. Si chaque groupe dogon semble puiser ses règles ou ses outils de classement dans un fonds culturel commun, l'agencement de ces éléments varie largement d'un village ou d'une région à l'autre, en s'adaptant à chaque contexte sociopolitique et en

Certains éléments de cette étude ont été exposés en 1996 et 1999 dans l'atelier «Âge et génération " du Laboratoire d'ethnologie et de sociologie comparative de l'Université Paris X-Nanterre. Par ailleurs, une première version de ce texte a été discutée dans l'atelier "Manuscrit en cours » du laboratoire Systèmes de pensée en Afrique noire. Je tiens à remercier pour leurs remarques les participants de ces ateliers. 
évoluant constamment pour des raisons démographiques ou historiques.

L'analyse des différentes formules adoptées par les Dogon permet ainsi de repenser l'articulation entre l'organisation d'âge et les institutions qui lui sont associées (société des masques, association de travail, système politique... ) ; elle apporte également un éclairage nouveau sur les rapports et les tensions entre classes d'âge, sexes et générations, ou entre âge, distribution du pouvoir et évolution individuelle.

Les Dogon, dont le nombre est estimé à 450 000, sont des agriculteurs établis à l'est du Mali, dans la bande soudano-sahélienne. Leur société, patrilinéaire et patrilocale, se caractérise par une organisation à la fois lignagère et villageoise. Chaque village est constitué de quartiers compacts et généralement contigus correspondant à autant de lignages exogames. Une moyenne de 450 personnes y réside mais, d'une localité à l'autre, les effectifs peuvent varier d'une centaine d'habitants à plusieurs milliers. Cette dimension lignagère et villageoise, plus ou moins marquée, subsiste même lorsqu'elle se combine localement à des formes d'organisation politique englobant plusieurs villages, notamment dans le cas des chefferies tene ou des fédérations tomo. Pour éviter des répétitions et des longueurs inutiles, je présenterai avant tout le versant masculin des différentes organisations d'âge dogon, en examinant successivement les cinq exemples retenus, puis j'aborderai en annexe leur pendant féminin. Dans les deux cas, je partirai d'une description relativement complète de la formule tenje, avant de décliner les autres plus succinctement, en mettant l'accent sur les différences.

\section{L'organisation d'âge tçฺe}

\section{Intervalles d'âge et espace intergénésique}

Pour aborder l'organisation d'âge tıne, il faut remonter à une des premières étapes du cycle de vie : l'imposition du nom. À Konsogou-donyou et dans tous les villages de Guimini, ce rite d'insertion lignagère a lieu une fois par an, juste avant le début de la saison des pluies, le troisième ou le quatrième jour de la fête du $a g u^{1}$. Les garçons et les filles nés au cours de l'année précédente sont rassemblés devant la maison de fonction du doyen de leur lignage. Des parents utérins rasent la tête des enfants rangés par ordre de naissance ; et deux vieilles accroupies de part et d'autre du seuil de la maison se passent chaque nourrisson, dans le même ordre, en le faisant entrer et sortir trois fois pour un garçon, quatre fois pour une fille. L'aînée des deux femmes pose sur un fer de houe le pied gauche du garçon ou le pied droit de la fille puis elle fait goûter au nouvel «entrant» la bière consommée les jours précédents par

1. Les termes dogon mentionnés dans l’article correspondent au parler de la région examinée. Pour des raisons typographiques, les signes $\varepsilon$, ว et $\mathrm{n}$ ont été transcrits è, ò et ng dans la carte et les tableaux. 


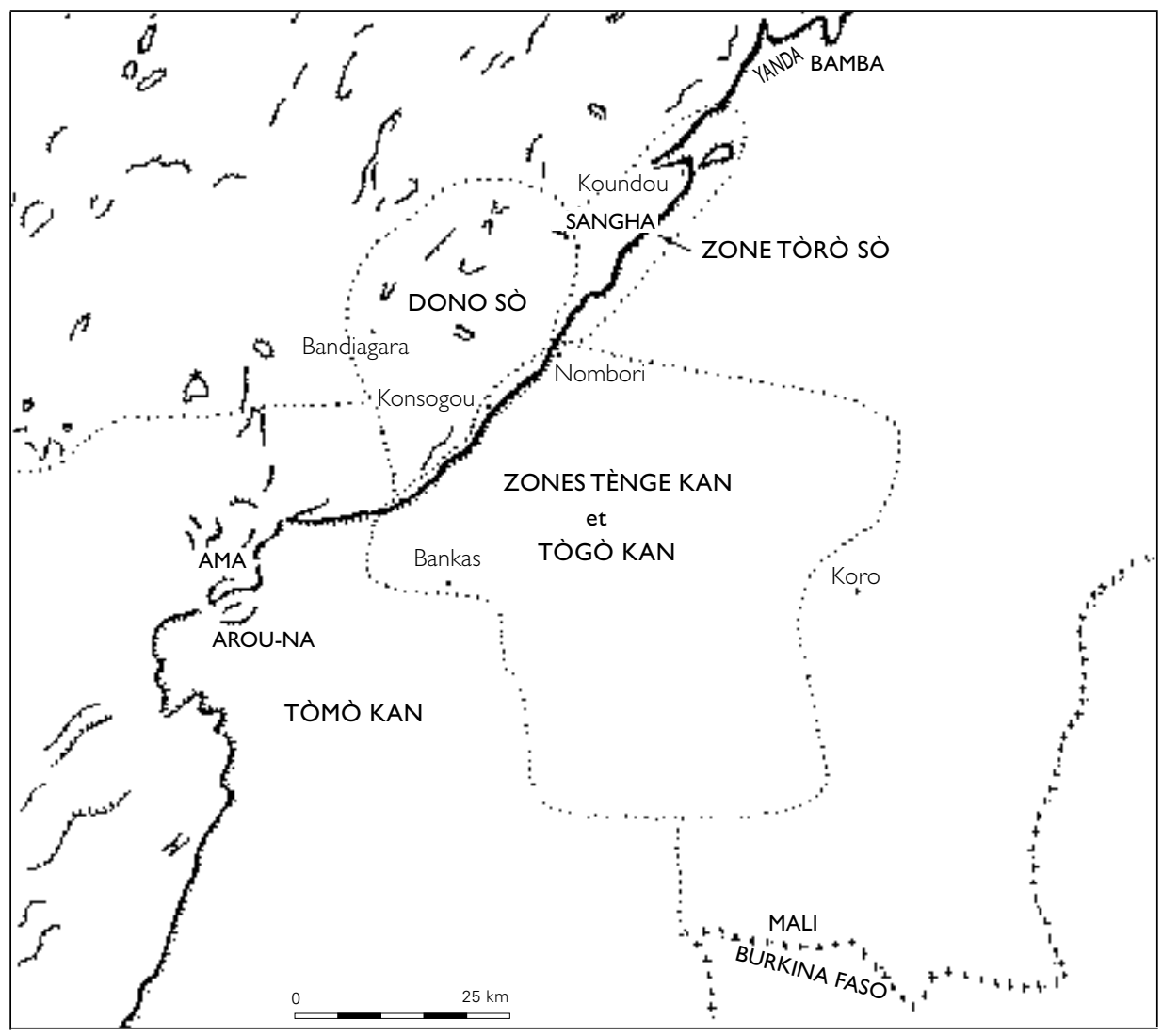

Régions dogon citées

les membres du lignage. Au même moment, le doyen attribue le prénom de l'enfant en fonction de son ordre de naissance au sein de sa fratrie. Cette cérémonie est la première opération de socialisation mais aussi de classement des individus : elle introduit chaque enfant dans son lignage et lui confere une identité sociale tout en lui attribuant un rang de naissance, aussi bien par rapport à ses camarades d'âge que par rapport à ses frères aînés.

La cohorte d'agnats nés à moins d'un an d'intervalle n'a pourtant rien d'une classe d'âge. Le rite de passage qui façonne leur identité se limite au cadre lignager et procède uniquement par rangement et agrégation; il ne sépare ni les frères ni les garçons et les filles, même si la distinction entre les sexes apparaît nettement à travers les gestes rituels et certains contournements de la règle de primogéniture ${ }^{2}$. En outre, cette "promotion " annuelle de nourrissons, appelée gaaru-gaaru, ne se positionne pas par rapport à un

2. Calqué sur la succession des naissances, l'ordre d'attribution des noms est théoriquement indépendant du sexe mais cette règle souffre une exception notable: le premier nourrisson à être introduit dans le lignage doit être nécessairement l'aîné des garçons. Si une fille le précède, elle sera nommée et donc rangée juste après lui, devenant de ce fait sa cadette pour respecter l'ordre de préséance entre les deux sexes. 
groupe aîné et cadet; elle s'inscrit dans une simple logique d'incorporation

lignagère: les derniers-nés, nommés et accueillis par les hommes et les femmes les plus âgés, rejoignent l'ensemble de leur communauté, en buvant la bière consommée avant eux par tous les membres de leur lignage.

Le terme gaaru-gaaru - « ceux de l'année » - sert à désigner l'ensemble des individus des deux sexes venus au monde dans le même intervalle, entre le début de la saison pluvieuse et la fin de la saison sèche suivante. Purement temporelle, cette délimitation ne s'applique pas à un contour spatial précis : qu'ils habitent le même village ou des localités voisines, deux hommes nés la même année sont gaaru-gaaru. Et ils sont gigiri s'il ont moins de trois ans de différence d'âge. Ce terme fait lui aussi référence à un intervalle et non à un groupe d'âge défini et stable ${ }^{3}$, mais, dans l'absolu, un gigiri comprend trois gaaru-gaaru successifs, soit les individus nés la même année et leurs cadets des deux années suivantes (tous gigiri les uns par rapport aux autres). Ces tranches d'âge de trois ans servent d'unité de mesure, notamment pour repérer l'époque où les enfants doivent être excisés ou circoncis. À Konsogou, par exemple, un garçon est en âge d'être circoncis lorsqu'il peut compter quatre gigiri après lui, c'est-à-dire lorsqu'il a entre 12 et 14 ans.

Du point de vue dogon, un gigiri correspond à un écart intergénésique optimal, en l'occurrence un intervalle de trois ans entre un frère ou une sœur et son cadet immédiat, appelé lui aussi gigiri (Kervran 1993 : 165 ; CalameGriaule 1968 : 103). À l'origine, cet espacement idéal était-il le résultat de la prohibition des rapports sexuels pendant la période de l'allaitement ? Denise Paulme (1940: 439-440) et Geneviève Calame-Griaule (1965: 336) évoquaient prudemment une telle règle en reproduisant les déclarations de leurs interlocuteurs dogon. Mais ces deux auteurs semblaient finalement douter, à juste titre, de l'existence d'une stricte interdiction des relations sexuelles jusqu'au sevrage; et certains de leurs informateurs avançaient d'ailleurs une autre explication de l'écart intergénésique, plus proche du discours actuel. Selon les conceptions dogon, le risque d'une nouvelle grossesse est naturellement plus faible pendant les deux années de l'allaitement - ce qui est loin d'être faux ${ }^{4}$ - et les femmes sont censées user de précautions supplémentaires pour éviter de tomber enceinte durant cette période, sans pour autant cesser les rapports sexuels. Un intervalle de trois ans, archétype du gigiri, sépare donc un frère et son cadet immédiat, en théorie du moins car, dans les faits, des naissances plus rapprochées ne sont pas rares.

3. Même si elle n'est pas une spécialiste des Dogon, Françoise Héritier avait repéré et défini cette notion de gigiri (gire en parler toro sว) grâce à une courte enquête menée à Sangha (1981 : 53-54).

4. La baisse de la fécondité pendant la période de l'allaitement est un phénomène bien connu (Peatrik 1999 : 487). 
Quelles sont les implications concrètes de ce modèle fondé sur l'espacement régulier des "frères germains"? S'ils appartiennent au même gigiri ou à deux gigiri adjacents, c'est-à-dire s'ils ont moins de six ans de différence d'âge, deux Dogon s'appellent mutuellement par leurs prénoms (Héritier 1981 : 54). En revanche, au-delà de deux gigiri, le cadet appelle son aîné de et ce terme d'adresse spécifique marque l'introduction d'un rapport d'aînesse incompatible avec une relation égalitaire. Voilà pourquoi deux amis intimes, ou les membres d'une même classe d'âge, se suivent forcément à moins de six ans d'intervalle. Cette règle ne signifie pas que les classes d'âge dogon se construisent en réunissant tous les hommes appartenant strictement à un ou deux gigiri. Unité de mesure et non principe de classement, l'écart intergénésique n'est qu'un des éléments pris en compte au moment du recrutement des classes d'âge. Il permet à chacun de calculer l'intervalle qui le sépare des autres, soit pour évaluer son âge, soit pour identifier ses aînés et ses cadets.

\section{Les promotions de circoncis}

En région tene, dans un village comme Konsogou-donyou, la cérémonie de circoncision est organisée théoriquement tous les deux ans en alternance avec le rituel d'excision, pour éviter que les filles et les garçons soient opérés la même année. Mais cette régularité est toute relative : la circoncision d'un groupe de garçons peut être reportée d'un an, voire décalée exceptionnellement de deux ans, à la suite d'une sécheresse, d'une épidémie ou d'un nombre trop restreint de "candidats». Par ailleurs, une fois la date de la cérémonie fixée, les pères disposent d'une certaine marge de liberté pour décider si leur fils âgé de 12 à 14 ans fera partie ou non de la prochaine promotion de circoncis. Un jeune garçon jugé trop chétif pourra ainsi être écarté alors qu'on anticipera volontiers la circoncision d'un enfant plus jeune mais physiquement plus développé ou présentant les premiers signes de la puberté. En outre, conformément à une règle en vigueur dans la plupart des systèmes de classes d'âge, deux frères ne peuvent être circoncis en même temps, a fortiori s'il s'agit de germains.

Si ces prescriptions empêchent un "découpage" des promotions par strict intervalle d'âge, elles aboutissent bien au résultat recherché : former au sein du village un groupe d' "alter ego " de sexe, d'âge et de corpulence identiques, se définissant entre eux par un rapport non pas d'aînesse mais de parité. Cela ne signifie pas que les positions d'aîné et de benjamin soient abolies à l'intérieur d'une classe de circoncis, mais elles ne fondent pas des rapports de domination ou de subordination. Les garçons circoncis ensemble sont statutairement égaux et leur initiation collective les dote d'une identité commune; ils deviennent ane, c'est-à-dire camarades d'âge 
et pairs, liés pour la vie par des devoirs d'entraide et de solidarité ${ }^{\text {. Premier }}$ échelon de l'initiation masculine et porte d'entrée sur l'âge adulte, la circoncision donne ainsi naissance à des promotions successives qui se superposent et s'apparentent à des classes d'âge (aye unu). Précisons que ces différentes promotions ne portent pas de noms distinctifs, et pour en désigner une en particulier le locuteur précisera simplement que c'est la classe d'âge d'untel, en nommant de préférence l'aîné du groupe.

Il n'est pas utile, pour la compréhension du système de classes d'âge tøne, de décrire en détail les rites de circoncision; la présentation de leurs caractéristiques les plus significatives suffira. Il s'agit schématiquement d'un rite de passage et d'une initiation se déroulant à l'échelle villageoise et transformant l'enfant prépubère en jeune homme adulte (sagatoro). Les garçons appartenant pour partie au même intervalle d'âge (gigiri) sont opérés par ordre d'âge décroissant, tous lignages confondus, puis ils subissent une retraite de 35 à 40 jours sous la surveillance et la férule des jeunes de la promotion précédente. Ces derniers exigent des novices nouvellement circoncis (konu unu) une obéissance absolue et ils ne sont pas avares, à l'occasion, de brimades ou de punitions. Ils leur apprennent des chants spécifiques, complètent rapidement leur éducation sexuelle, leur rase la tête à la fin de leur réclusion et enfin s'approprient une partie des dons reçus par les nouveaux circoncis. Ceux-ci, une fois libérés, ne repartent pas habiter chez leurs parents mais occupent à plusieurs une maison de célibataires (guj). Deux ou trois ans plus tard, ils surveilleront à leur tour les membres d'une nouvelle promotion de circoncis, et ce n'est qu'après cette étape qu'ils entreront de plein droit dans l'association de culture des jeunes. Chaque groupe de circoncis se construit ainsi, dès les premières années de sa création, en liaison avec les deux promotions adjacentes : l'aînée et la cadette. Prêtons attention toutefois au sens des termes aîné et cadet : dans cette configuration, et du point de vue dogon, ils traduisent davantage un rapport de précédence qu'une relation d'aînesse pleinement réalisée. Rappelons que les membres de trois promotions successives ne sont séparés que par un intervalle de six ans; ce qui exclut entre eux toute relation d'aînesse fortement marquée. Nous verrons en revanche que la distinction "aîné/cadet " réapparaît formellement entre des groupes de travail englobant chacun trois promotions de circoncis.

Avant de passer à l'association de culture, il convient de nuancer l'équivalence posée initialement entre promotion de circoncis et classe d'âge.

5. L'expression «fraternité d'âge » adoptée notamment par Denise Paulme (1940: 236) renvoyait bien sûr à ces liens de solidarité, mais elle introduisait une ambiguïté en assimilant les membres d'une même promotion de circoncis à des " frères ", alors que ces derniers se répartissent justement entre des promotions différentes. D'ailleurs, D. Paulme (1968) critiquera et abandonnera par la suite ce terme de "fraternité». 
Pour des raisons évoquées précédemment (maladie, absence, scolarité, complexion malingre ou puberté précoce), il arrive que plusieurs garçons soient dissociés de leurs camarades d'âge et circoncis séparément, un an après ou avant, soit dans un autre village (en étant alors ajoutés aux enfants de cette localité), soit, plus rarement, dans un quartier différent du même village. Dans ce cas, les circoncis nés approximativement dans le même intervalle de deux ans se trouvent répartis dans deux promotions différentes. Mais dès qu'ils rejoignent l'association de travail, ces deux groupes vont fusionner pour ne former en définitive qu'une seule classe d'âge ${ }^{6}$. Un tel ajustement est observable à Konsogou-donyou pour les classes de rang 0 et 5, avec une «session » de rattrapage en 1995 et une circoncision anticipée dans le village voisin de Konsogou-tomoro en 1981 (cf. tableau 1).

\section{L'association de travail et le grade d'âge des jeunes gens}

En région tene, à Konsogou-donyou, l'association de culture (wana mosnu) se charge essentiellement de battre le fonio des différents chefs de famille, au moment de la récolte. Tous les jeunes gens de 15 à 37 ans appartiennent à cette association villageoise qui regroupe, selon les décomptes, de neuf à onze classes d'âge réparties sur autant de degrés. À chaque fois qu'une nouvelle promotion de circoncis entre, toutes les autres se décalent d'un rang et poussent ainsi la plus ancienne vers la sortie. Mais l'entrée comme la sortie s'effectuent en deux temps, par le biais d'un échelon intermédiaire situé à chaque extrémité de ce groupe de travail.

C'est le cas, on l'a évoqué, pour les nouveaux circoncis : ils ne se rattachent pleinement à l'âge adulte et à l'association de culture qu'après avoir exercé leur autorité vis-à-vis de la promotion suivante. Entre temps, ils ont été dispensés des travaux les plus éprouvants pour se voir confier certains préparatifs particuliers, comme le nettoyage de l'aire de battage. Mais ce traitement de faveur n'explique pas le paradoxe suivant: les membres de la dernière promotion de circoncis participent concrètement à la plupart des activités de l'association de travail tout en étant considérés, au niveau du discours, comme des "novices" plus ou moins extérieurs au groupe. Ce paradoxe ne peut s'expliquer que par la nature même de cette association, conçue non comme un groupe d'âge fermé sur lui-même mais comme un ensemble de classes d'âge occupant chacune une position d'aîné et de cadet. En tant que benjamins absolus, les nouveaux circoncis sont relégués fictivement hors de l'association de travail, ou du moins à sa marge, et ils doivent attendre la formation d'une classe "cadette " pour 6. Les deux promotions continuent d'exister dans la mémoire de ses membres mais ces derniers ne constituent plus, statutairement, qu'un seul et même groupe de travailleurs, de commensaux, de prestataires... 
occuper pleinement leur place dans ce système d'échelons où chaque promotion est liée à la précédente et à la suivante.

La même logique s'applique, avec plus de netteté, à l'extrémité supérieure de l'association de travail. Au sein de ce groupe, la plus ancienne des classes d'âge se situe au neuvième rang, si l'on exclut justement la dernière promotion de circoncis. L'aîné de cette classe est donc le doyen, mais non le chef, de l'ensemble de l'association de travail. Les membres de sa promotion ont déjà amorcé un timide retrait: s'ils continuent de battre le fonio de nuit, ils s'abstiennent désormais de le faire pendant la journée, en limitant leur action au ramassage de la paille sur l'aire de battage. À l'échelon supérieur, la promotion précédente a quitté officiellement l'association de travail tout en conservant certains liens avec elle. Elle a demandé et reçu l'autorisation formelle de partir après l'incorporation d'une nouvelle promotion de circoncis; de ce fait, elle ne participe plus au battage du fonio mais intervient néanmoins pour ramasser la paille pendant les travaux nocturnes. La promotion qui occupe cet échelon porte d'ailleurs le nom de «ramasseurs de paille de fonio » (pono jija sono we), en référence à leur seule

\begin{tabular}{|c|c|c|c|c|}
\hline Année de circoncision & Membres de l'association de travail & Masques portés & Groupes & Catégories d'âges \\
\hline $\begin{array}{l}10(1972) \\
\pm 37-38 \text { ans }\end{array}$ & $1-2-3-4-5-6-7$ & & $\begin{array}{l}\text { pongo jija sònò we } \\
\text { « ceux qui ramassent } \\
\text { la paille de fonio » }\end{array}$ & $\begin{array}{l}\text { sagatòrò woroju } \\
\text { « jeune homme qui } \\
\text { s'arrache » ( } \pm 37 \text { ans })\end{array}$ \\
\hline $\begin{array}{l}9(1974) \\
\pm 35-36 \text { ans }\end{array}$ & \multicolumn{2}{|l|}{$\begin{array}{l}\underline{8} \text { (aîné de l'association de travail) } \\
-9-10-11\end{array}$} & \multirow{3}{*}{$\begin{array}{l}\text { ange-unu dere } \\
\text { " groupe d'âge aîné » }\end{array}$} & \multirow{3}{*}{$\begin{array}{c}\text { sagatòrò kunyu } \\
\text { « jeune homme } \\
\text { rugueux » ( } \pm 29-36 \text { ans })\end{array}$} \\
\hline $\begin{array}{l}8(1976) \\
\pm 33-34 \text { ans }\end{array}$ & \multicolumn{2}{|l|}{$12-13-14-15-16$} & & \\
\hline $\begin{array}{l}7(1978) \\
\pm 31-32 \text { ans }\end{array}$ & \multicolumn{2}{|l|}{$17-18-19-20-21-22-23$} & & \\
\hline $\begin{array}{l}6(1980) \\
\pm 29-30 \text { ans } \\
+\quad 20 \ldots \ldots \ldots \ldots\end{array}$ & \multicolumn{2}{|l|}{$24-25-26-27-28-29-30$} & \multirow{4}{*}{$\begin{array}{c}\text { ange-unu sugòn } \\
\text { " groupe d'âge cadet » }\end{array}$} & \multirow{4}{*}{$\begin{array}{c}\text { sagatòrò } i \\
\text { «petit jeune homme » } \\
( \pm 20-28 \text { ans })\end{array}$} \\
\hline 5bis $(198 I) \pm 27-28$ ans & \multirow{2}{*}{\multicolumn{2}{|c|}{$\begin{array}{l}31-32 \text { - } 33 \text { (circoncis à Konsogou-tomoro) } \\
34-35-36\end{array}$}} & & \\
\hline $5(1982) \pm 26-27$ ans & & & & \\
\hline $\begin{array}{l}4(1985) \\
\pm 22-25 \text { ans }\end{array}$ & \multicolumn{2}{|l|}{$\begin{array}{l}37-38-39-40-41-42-43-44- \\
45-46\end{array}$} & & \\
\hline $\begin{array}{l}3(1986) \\
\pm 20-21 \text { ans }\end{array}$ & $\begin{array}{l}\frac{47}{50} \text { (buru dinè banga) - } 48-49- \\
\text { (circoncis à Konsogou-tomoro) }\end{array}$ & $\begin{array}{l}\text { masque bèrè-bèrè en } \\
\text { fibres brutes et teintes }\end{array}$ & \multirow{3}{*}{$\begin{array}{l}\text { ange-unu dunò } \\
\text { "groupe d'âge } \\
\text { benjamin » }\end{array}$} & \\
\hline $\begin{array}{l}2(1990) \\
\pm 17-20 \text { ans } \\
\end{array}$ & $\begin{array}{l}51-52-53-54-\underline{55-56} \\
(« \text { accompagnateurs de jeunes filles })\end{array}$ & $\begin{array}{l}\text { masque ajakay en } \\
\text { fibres brutes }\end{array}$ & & \multirow{3}{*}{$\begin{array}{c}\text { sagatòrò } \boldsymbol{i} \text { dagi } \\
\text { " tout petit jeune } \\
\text { homme » } \\
( \pm 12-19 \text { ans })\end{array}$} \\
\hline $\begin{array}{l}1 \text { (1992) } \\
\pm 15-16 \text { ans }\end{array}$ & $\begin{array}{l}57-58-59-60-61-62-63- \\
64-65-66-67\end{array}$ & $\begin{array}{l}\text { masque sanangurèy } \\
\text { en feuilles }\end{array}$ & & \\
\hline $\begin{array}{l}\text { Obis }(1994) \pm 13-14 \text { ans } \\
0 \text { (1995) } \pm 12-13 \text { ans }\end{array}$ & \multicolumn{2}{|c|}{$\begin{array}{l}68-69-70-71 \text { (quartier du bas) } \\
72-73-74-75-76-77 \text { (quartier du haut) }\end{array}$} & $\begin{array}{c}\text { kònu unu } \\
\text { nouveaux circoncis }\end{array}$ & \\
\hline
\end{tabular}

Tableau I Les différentes promotions de circoncis formant l'association de travail (village tenge de Konsogou-donyou, en juin 1995) 
activité agricole commune. Lexistence de cette promotion supérieure, repoussée à la marge, permet à la classe aînée de l'association de travail de s'insérer dans une relation sur trois niveaux.

À l'intérieur de l'association de culture, les regroupements s'opèrent selon la même division ternaire. Les classes d'âge juvéniles ne travaillent jamais séparément : soit elles interviennent toutes ensemble, notamment dans de petits villages comme Konsogou-donyou, soit elles se rassemblent par groupe de trois, en particulier lorsqu'il s'agit de sarcler les champs du beaupère de l'un des jeunes. L'intervalle d'âge explique encore une fois cette triade laborieuse : les membres de trois promotions successives ont six ans de différence d'âge et, à ce titre, ils forment un groupe solidaire où l'entraide et la réciprocité sont la règle. Toute association de culture tøne est d'ailleurs subdivisée en trois sections comptant chacune trois promotions de circoncis et portant le même nom que n'importe quel groupe d'âge (ane unu). Dans les gros villages, ces trois sections peuvent être mobilisées séparément, suivant la nature et l'ampleur de la tâche à accomplir. À Konsogou, en revanche, ce type de subdivision ternaire répond plus à un besoin d'ordonnancement qu’à une nécessité pratique. Qu'elles correspondent ou non à de véritables groupes de main-d'œuvre, ces sections de travail présentent deux différences notables par rapport aux promotions de circoncis. Les rapports d'aînesse qui les lient deux à deux s'expriment ouvertement à travers les noms qu'on leur attribue : groupe aîné (ane unu dere), cadet (-sugon) et benjamin (-duns). En outre, elles se renouvellent par tiers tous les deux ans, à chaque nouvelle promotion de circoncis.

Jusqu'au troisième échelon de l'association de travail, la progression d'une même classe d'âge s'accompagne d'une élaboration croissante des masques portés par les jeunes gens. Les premiers échelons, définis après la circoncision, sont en effet communs à l'association de culture et à une société des masques autrefois embryonnaire et aujourd'hui en cours de déliquescence. Dans l'année qui suit la circoncision, au moment du rituel appelé sanayurcy-segu, les garçons offraient à leurs aînés des deux promotions précédentes des noyaux de fruits de Sclerocarya birrea, dont l'amande est comestible. Cette prestation était le prix à payer pour «approcher» les masques sananurcy en feuilles de Lannea microcarpa. À cet échelon, que ce soit dans le cadre de la société des masques ou de l'association de travail, les nouveaux circoncis sont considérés comme des "novices » et ils se contentent d'assister à la fabrication des sananurcy par les aînés de la promotion précédente ; ils les aident également à couper des branches et à s'en couvrir.

Deux ans plus tard, après la formation d'une nouvelle promotion de circoncis, ces mêmes jeunes portent à leur tour un masque en feuilles, alors qu'ils ont déjà rejoint l'association de travail. Ils patrouillent en brousse et 
dans le village deux années de suite, un peu avant la saison des pluies, le corps recouvert de feuillage. Quand leur classe d'âge avance d'un rang, ils revêtent un masque ajakay en fibres brutes, confectionné avec l'écorce du même arbre (Lannea microcarpa). Enfin, à l'échelon suivant, ils ont le droit de porter un bere-bere, composé d'une tête de ajakay et d'une jupe en fibres teintes. Précisons que les rites d'incorporation à la société des masques ont disparu à Konsogou en 1985, mais les jeunes continuent de revêtir successivement les mêmes masques - sanayurcy, ajakay, bere-bere - aux trois premiers échelons de l'association de travail. Au moment où les garçons sont en plein développement physique, leurs masques se transforment simultanément à chaque franchissement d'échelon, en évoluant du plus simple au plus complexe, du plus petit au plus grand, de la matière brute à la forme la plus travaillée. Depuis la transformation corporelle introduite par la circoncision, le corps des garçons et le masque qu'ils portent vont donc évoluer conjointement, même si des adaptations seront parfois nécessaires ; il arrive par exemple qu'un jeune d'apparence trop frêle attende d'avoir suffisamment forci pour revêtir le robuste masque ajakay.

Laissons désormais de côté la structure interne de l'association de culture pour ne considérer cette fois que le groupe d'âge qu'elle représente. Composée d'un nombre invariable de classes d'âge échelonnées depuis la circoncision jusqu'aux "ramasseurs de paille de fonio", l'association regroupe l'ensemble des "jeunes hommes » et délimite la génération des " fils " ${ }^{7}$. En effet, que ce soit en théorie ou en pratique, ce système est construit pour qu'un homme ait déjà quitté ce groupe de travail quand son fils y entre à son tour. Entre l'aîné et le benjamin des travailleurs, l'écart d'âge semble calculé au plus juste: il est de dix-huit ans en théorie et de vingt-et-un ans dans l'exemple examiné ici, si bien qu'en 1994, à Konsogoudonyou, l'aîné des «ramasseurs de paille de fonio » est sorti de l'association de travail à 37 ans, au moment où son fils aîné rejoignait le groupe à 15 ans. Cette règle d'évitement générationnel est d'ailleurs valable pour l'ensemble du pays dogon, et énoncée comme telle : un fils et un père ne doivent jamais se retrouver dans la même association de travail. La dissociation des pères et des fils, mais aussi la séparation des frères, répartis dans des classes différentes, organisent ainsi l'association de travail en une fratrie de « fils » appartenant par définition à une même génération (ba-unu).

La correspondance entre cette génération des fils et la classe des « jeunes hommes » exige quelques précisions. Au moment de la circoncision, la coïncidence est quasiment parfaite : le garçon quitte le monde de l'enfance et la tutelle maternelle pour accéder avec toute sa promotion au grade des

7. Le terme de "génération » est employé ici pour désigner un rapport de filiation fictif, hors de la sphère de parenté, entre tous les hommes du village séparés par un même intervalle d'âge. 
" jeunes» (sagatsro), en devenant d'abord un "très petit jeune homme» (sagator $i$ dagi). Simultanément, il entre dans la génération des fils, même si, en tant que nouveau circoncis, il se maintient deux ans dans une période intermédiaire. En sortant de l'association de travail, vers 37 ans, il change encore de catégorie d'âge: il devient sagators woroju, expression qui désigne littéralement un «jeune homme qui s'arrache", en "se détachant " aussi bien de l'association de travail que de la génération des fils et du grade des « jeunes» (cf. tableau 2).

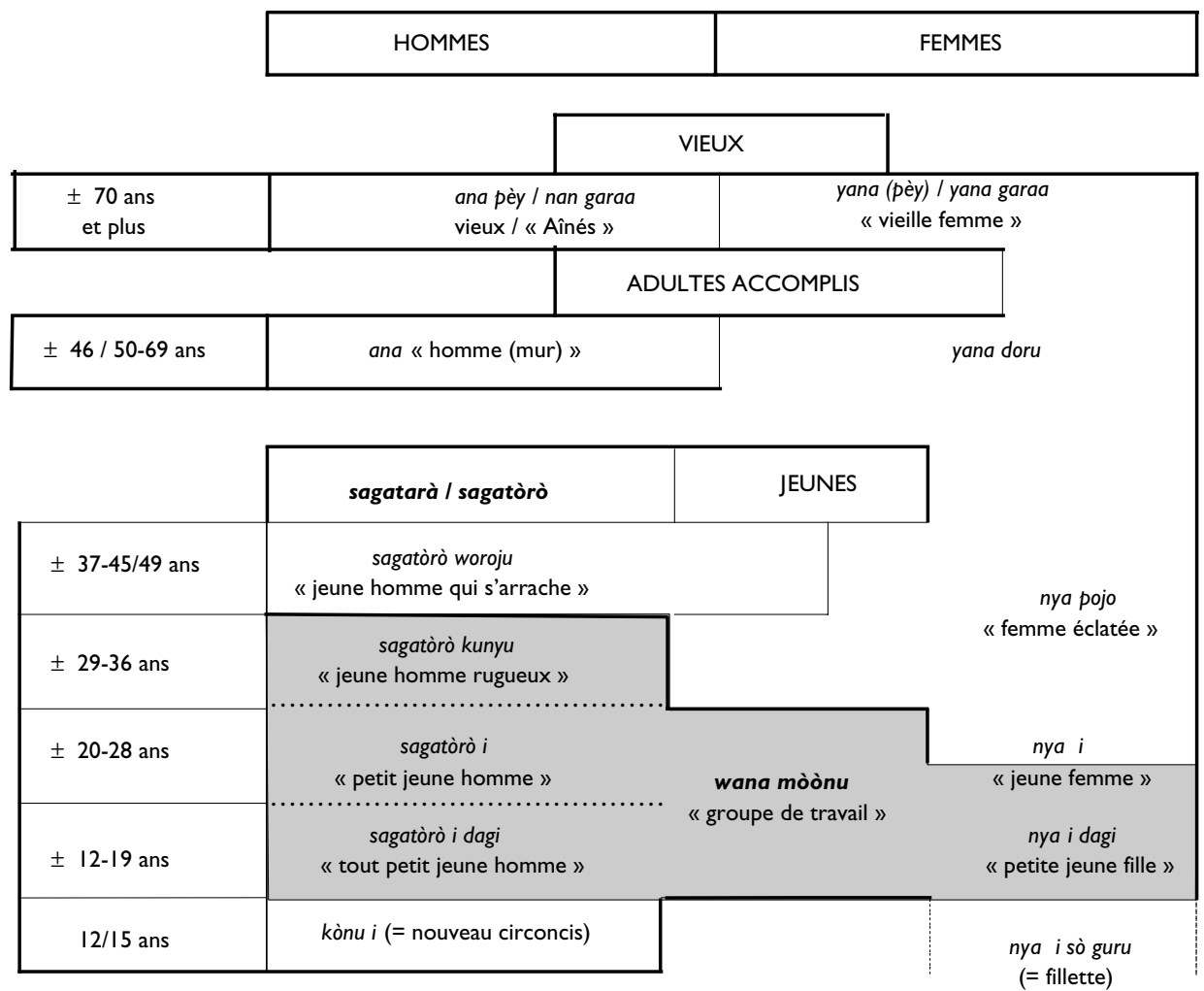

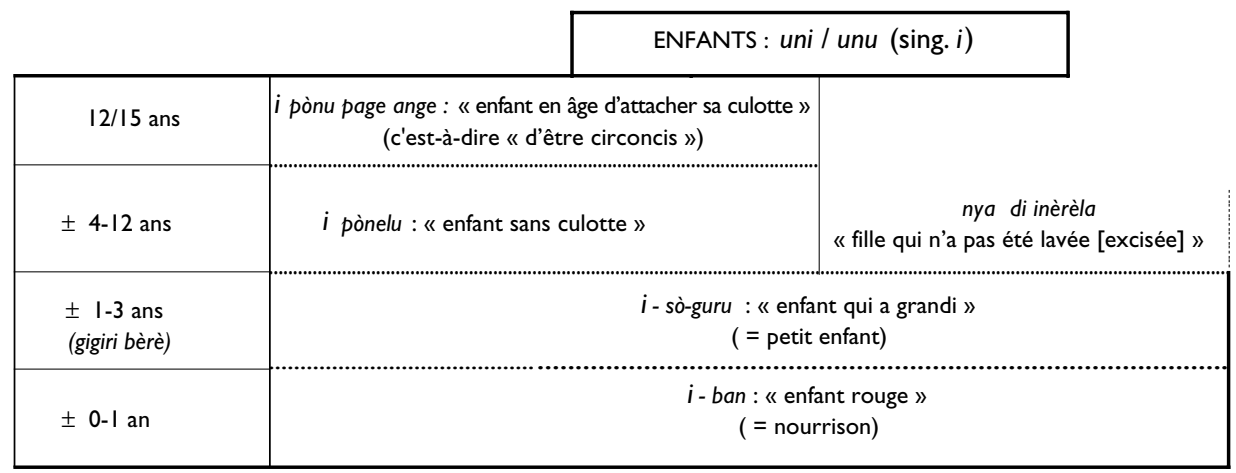

Tableau 2 Catégories d’âge (parler t乏̣̌e kan de la région dogon de Guimini) 
Ce degré d'âge fait office d'échelon intermédiaire et permet de passer d'une position générationnelle à la suivante. En effet, une fois libérés de l'association de travail, les membres d'une classe d'âge ne deviennent pas pour autant des "hommes » et des " pères "; ils doivent attendre que leurs enfants prennent leur place à la génération des "fils ", après avoir été circoncis. Dans la logique de ces générations qui se construisent sur un mode ascendant, ce sont en quelque sorte les fils qui "engendrent" les pères, après les avoir poussés hors de l'association de travail et de la classe des "jeunes». En dehors de cette perspective générationnelle, la catégorie des "hommes" (ana) est beaucoup plus floue; ses contours fluctuent selon la position du locuteur par rapport à la personne qu'il désigne sous ce vocable: un même individu peut donc être sagators woroju pour un homme et ana pour un autre. Cette relativité ne disparaît que si on se place justement du point de vue des fils : les membres d'une même promotion passent ipso facto dans la catégorie des "hommes (accomplis)", pour leurs fils, dès que ces derniers deviennent eux-mêmes des "jeunes hommes". La période de transition et de "détachement" des sagator woroju semble donc aménagée dans le seul but de faire coïncider la classe des "hommes" à la génération des pères. Dans la formule tene, un homme peut ainsi se retrouver entre deux âges et entre deux générations alors que cette situation est évidemment impossible dans un système générationnel (Peatrik 1999: 18).

Quant aux autres subdivisions de la catégorie "jeune", aucune ne correspond vraiment à un échelon statutaire ou à un nombre déterminé de classes d'âge, si bien que leur délimitation reste imprécise et dépend pour une part de l'appréciation de chacun. Sagator $i$ dagi, sagators $i$ et sagatsro kunyu sont des catégories d'âge relatives et deux individus différents pourront classer un jeune dans deux catégories voisines, en fonction des informations qu'ils détiennent et des critères qu'ils vont privilégier: âge réel, développement physique, rang de sa classe d'âge ou statut matrimonial. Il est vrai qu'autrefois les âges étaient associés plus étroitement aux étapes matrimoniales. Garçons à peine pubères, les "très petits jeunes hommes " vivaient à plusieurs dans une maison de célibataires et n'avaient pas encore commencé à courtiser les filles. Les "petits jeunes hommes ", en revanche, avaient leur propre maison, construite dans le quartier de leur père, et ils étaient engagés dans des relations prémaritales tout en étant redevables de certaines prestations à leurs futurs beaux-parents. Enfin, les "jeunes hommes rugueux" (sagatsro kunyu) étaient des hommes mariés habitant avec leur épouse, mais ils n'avaient pas renoncé pour autant aux flirts extraconjugaux et traînaient derrière eux une réputation de maris volages. La situation a toutefois changé : le mariage est de plus en plus précoce et ces 
étapes matrimoniales sont aujourd'hui décalées par rapport aux degrés d'âge. Les jeunes d'une même classe d'âge continuent malgré tout à franchir ensemble chacune de ces étapes, la même année, à la suite de leurs aînés. Par ailleurs, si l'on considère cette fois l'ensemble des « jeunes ", ceuxci se caractérisent toujours par leur propension à courtiser les femmes, par opposition au statut ultérieur des " hommes ", pères et maris accomplis.

Comment se distribuent les charges au sein de l'association de culture? Le véritable responsable est le buru dine baya, "celui qui a la garde de la trompe (pour appeler au travail)». Les jeunes le choisissent en leur sein tous les deux ans ${ }^{8}$, en fonction de ses compétences mais aussi de sa famille, pour que la charge passe à tour de rôle dans les différents lignages et segments de lignage constitutifs du village. Il s'agit généralement d’un " petit jeune homme" d'une vingtaine d'années; il programme les travaux collectifs, négocie la contrepartie alimentaire ou financière, puis récupère et épargne les sommes dues. Son adjoint et successeur, chargé de la distribution de la crème de mil au cours des travaux collectifs, est choisi dans la classe d'âge suivante. À Konsogou-donyou, à chaque changement du "gardien de la trompe ", deux garçons sont également désignés pour être les "accompagnateurs de jeunes filles» (nya unu niine) lors des travaux nocturnes; ils appartiennent aux deux lignages du village et à une des classes d'âge les plus jeunes. Enfin, tous les deux ans, dans un lignage à chaque fois différent, les membres de l'association de travail choisissent un chef purement honorifique parmi les hommes d'âge mûr et les "pères " de leur village. Ce dernier prend le nom de mar'pa uni amiri, "chef des fusiliers ". Contrairement à ce qu'on pourrait croire, un tel titre ne fait pas référence à un éventuel rôle militaire de l'association de travail mais à sa dimension festive : à l'occasion de la fête de fin d'année, les jeunes partent en cortège saluer leur "chef» en brandissant leurs fusils et en tirant des salves d'honneur et de réjouissances; l'homme les reçoit avec de la bière et de la viande offertes en contrepartie des travaux effectués à son bénéfice par les jeunes, en particulier le sarclage de son champ de mil.

Comme le montre le choix des différents responsables, l'association de culture compose avec l'organisation lignagère tout en endossant une dimension essentiellement villageoise. L'autorité au sein de ce groupe n'est jamais fondée sur le principe d'aînesse (qui structure l'organisation lignagère), et si le doyen du groupe joue malgré tout un certain rôle, il se contente de nommer les « accompagnateurs de jeunes filles» et de désigner

8. Le "gardien de la trompe " reste en activité deux ans mais seules sont comptabilisées les années où l'association de travail est sollicitée pour le battage collectif du fonio. 
formellement un nouveau "gardien de la trompe », lorsque le précédent lui a remis son mandat. Le choix de ce responsable et du "chef des fusiliers" s'inscrit d'ailleurs dans la logique du système politique tenje où le chef politico-religieux - le ogo waju - était nommé indépendamment du principe d'aînesse. Mais l'association de culture n'a jamais travaillé pour ce ogo waju, que ce soit pour cultiver ses champs de fonction ou pour assurer en son nom la police intérieure; elle n'avait aucun lien avec lui, pas plus qu'il n'avait de prise sur elle ou sur les classes d'âge qui la composent. En revanche, le lien entre l'association de culture et le chef honorifique qu'elle s'est choisie est particulièrement révélateur : en tant que groupe de travail, les jeunes se mettent au service des générations précédentes, dont ils reconnaissent l'autorité morale; et en tant que société de festin, ils convertissent en boisson les gains réalisés pour faire la fête ensemble, entre jeunes du même âge, dans un esprit de partage et d'égalité.

Les classes d'âge juvéniles, regroupées en association de travail, ne sont pas une menace pour l'ordre lignager ; elles apparaissent plutôt comme un complément à l'organisation lignagère et villageoise. D'ailleurs, dans certains villages tø̣e, l'apparition récente de «groupements villageois coopératifs" traduit l'affaiblissement de ce type d'organisation au profit de nouvelles solidarités. Ces groupements, fondés sur le volontariat, reposent souvent sur une base confessionnelle et rassemblent par exemple la majorité des catholiques d'une même localité. Ces derniers ne travaillent plus dès lors pour les aînés du village, mais pour eux-mêmes et les membres de leur communauté religieuse?.

\section{Les "pères" : des hommes accomplis}

Dispensées de travaux collectifs, les classes d'âge des plus de 40 ans se dégagent du système de regroupement ternaire propre à l'association de travail. Dès lors, l'appartenance à une promotion de rang $\mathrm{X}$ ou $\mathrm{Y}$ cesse d'être un élément important du statut individuel. En tant que chef de famille déjà bien établi dans le mariage, "l'homme " (ana) se replie sur son unité domestique en exerçant son rôle de père et de mari exemplaires; mais il est libre aussi de s'engager dans une activité personnelle lucrative ou valorisante: boucher, menuisier, barde itinérant, chasseur expérimenté, devin, etc. Ana est par excellence un âge intermédiaire où, statutairement, une certaine forme d'émergence individuelle est possible, après s'être débarrassé des corvées collectives assumées par les jeunes et avant d'endosser les responsabili-

9. Ces groupes de travail, dont le mode de recrutement ne reposait plus uniquement sur l'âge, étaient apparus après l'indépendance du Mali, au moment de l'expérience socialiste de Modibo Keita. Ce dernier s'était inspiré des associations de culture juvéniles pour développer des groupements coopératifs villageois, appelés Mali-Ton. 
tés rituelles dévolues aux anciens. Certains hommes d'une cinquantaine d'années partent d'ailleurs approfondir leurs connaissances dans des régions voisines pour devenir ainsi, à leur retour, des "personnes mûres " (nan ire), dans le sens d'hommes expérimentés, parvenus au sommet de leur art dans le domaine de la magie, de la cynégétique ou de la pharmacopée. Dans le contexte régional tøne, le grade des " hommes » et des " pères » est également celui où s'exerce le pouvoir, réel ou formel, des chefs : ogo-waju (chef politico-religieux choisi justement vers 45-50 ans), amiri (chef de village, nommé généralement à ce poste en tant que fils aîné du doyen d'un des lignages), mar'pa uni amiri (tuteur de l'association de travail), ou plus généralement chef de famille. De nature individuelle, ce pouvoir des " hommes » ou des "pères " s'oppose bien sûr à l'autorité collégiale des «vieux " et à la sujétion des " jeunes", soumis aux tâches collectives.

Au-delà de 40 ans, les membres d'une classe d'âge se réunissent uniquement pour célébrer les funérailles d'un des leurs ou pour préparer et boire ensemble de la bière, lors de certaines cérémonies villageoises. Dans ce cas, chacun fournit la même quantité de mil, conformément à la règle d'égalité qui prévaut au sein de ce groupe. Par exemple, lors du rituel annuel du nyere ${ }^{10}$, chaque classe d'âge des "hommes" fixe le nombre de mesures de mil réclamé à ses membres afin de rassembler les six à huit calebasses de grain nécessaires à la confection d'une grande jarre de bière. À tour de rôle, l'épouse d'un des camarades d'âge se charge de brasser la boisson. Une partie de la bière est apportée sur le lieu du rituel, pour être "goûtée " par les vieux, et l'autre partie est consommée par les membres de la classe d'âge. Pour la cérémonie du nyere, le nombre de " cabarets » de bière et celui des classes médianes devraient donc correspondre, du moins en théorie. Or, concrètement, c'est rarement le cas, surtout pour un petit village de la taille de Konsogou-donyou où, vers 50-60 ans, les classes d'âge ne comptent plus parfois qu'un ou deux représentants, en raison d'une forte émigration et d'un taux élevé de mortalité. L'effacement progressif des classes médianes et supérieures est d'ailleurs un phénomène démographique qui concerne tous les villages, si bien que plusieurs promotions successives, voire l'ensemble des « hommes », se regroupent généralement pour préparer ensemble un cabaret de bière.

Au bout du compte, le seul modèle qui s'impose dans tous les villages, c'est la séparation de chacune des trois générations lors des rituels. Ces trois groupes préparent et boivent la bière cérémonielle à des moments distincts, parfois sur trois jours différents : les "vieux ", principaux acteurs du rituel,

10. Équivalent du muns et du mooro des régions voisines, le nyere est un rituel annuel tęe célébré à la fin de la saison sèche pour assurer la protection et la fécondité du village. 
se rassemblent entre eux, en premier, sur le lieu du sacrifice ; les " hommes " se retrouvent ensuite pour boire, soit tous ensemble, soit par classe d'âge (sans hiérarchie apparente) ; enfin les « jeunes » interviennent en dernier, en tant que groupe de travail, après avoir transformé en bière les gains de leur association. Quant aux nouveaux circoncis, toujours situés à la marge, ils sont généralement exemptés de cotisations en mil mais ils apportent néanmoins le bois de cuisson pour la préparation de la bière.

\section{Les Vieux et les Anciens}

Ana pey, "vieux", est d'abord une catégorie d'âge aux contours parfois imprécis, au même titre que "jeune » ou "homme». Quelqu'un peut être qualifié de « vieux » au vue de sa seule apparence physique, mais il s'agit alors d'une appréciation purement formelle portée par exemple sur un inconnu. En revanche, un homme ne peut revendiquer le titre de "vieux» que s'il a acquis la dignité et le statut qui siéent à cet âge ; ce qui suppose son incorporation préalable au cercle des «Seniors » ou des «Anciens » $(\text { nan garaa })^{11}$, en charge des rituels villageois. Statutairement, être vieux, cela signifie «être assis » et siéger au sein d'une assemblée réunissant les hommes les plus âgés du village. L'expression nan daye, "ceux qui sont assis", est d'ailleurs la métaphore la plus courante pour désigner les Vieux du village; elle évoque leur posture réelle mais aussi leur assise sociale et leur autorité collégiale.

Si les classes d'âge juvéniles entrent, gravissent les échelons et sortent de l'association de travail en étant poussées par chaque nouvelle promotion de circoncis, l'accès au statut d'Ancien et au grade des « vieillards » obéit à un autre mode de recrutement. Ce passage a lieu vers 70 ans, mais il ne s'effectue pas en fonction du nombre de promotions que les Vieux ont derrière eux - ils seraient d'ailleurs incapables de les compter - ni même en raison d'un éventuel décalage automatique de ces promotions. Les habitants d'un même village rejoignent le groupe des Vieux en fonction du nombre d'aînés survivants qu'ils ont devant eux, ou, ce qui revient au même, en fonction des places laissées vacantes par les vieillards défunts. En effet, il faut toujours un effectif minimum voire un nombre fixe de Seniors responsables de l'exécution des rituels villageois. Afin de combler un vide relatif, l'aspiration par le sommet remplace donc la pression de la base; et ce sont d'ailleurs les Anciens qui contactent leurs cadets les plus proches pour tenter de les recruter.

11. Nan garaa signifie littéralement "personne(s) plus âgée(s); supérieure(s)». Ni le genre ni le pluriel ne sont marqués et la formule manquerait donc de précision si elle ne désignait, par convention, les Anciens ayant la charge du village, ou tout du moins de ses autels. L'expression aná garaa est plus ambiguë dans son usage courant ; elle se réfere soit à une aînesse relative - un homme plus âgé que soi -, soit à une aînesse absolue - un homme parmi les plus âgés - et elle est alors synonyme de ana pey. Quant à l'expression àna garaa, elle s'emploie spécifiquement pour le "doyen du village ». 
Une telle demande n'est pas forcément agréée dans l'immédiat: les "hommes " d'âge mûr ne renoncent pas facilement à leur liberté pour endosser les responsabilités contraignantes des Seniors, d'autant que leur nouvelle dignité de vieillard leur interdirait certaines licences admises à l'âge précédent, notamment dans le domaine de la boisson. L'entrée dans l'ultime grade d'âge des Vieux fait donc l'objet d'une négociation et l'homme ainsi sollicité peut tergiverser et temporiser pendant une ou deux années, mais il finit toujours par céder, en entraînant parfois dans son sillage son cadet, même si celui-ci appartient à une classe d'âge inférieure. Tous deux rejoignent alors physiquement l'assemblée des Anciens en participant avec eux aux rituels et en formant désormais une même communauté de buveurs; dès ce moment, ils doivent adopter une conduite digne et réservée conforme à leur nouveau statut. À ce stade, les classes d'âge se sont fondues d'autant plus facilement dans ce "club du troisième âge " que certaines d'entre elles ont déjà disparu avec l'ensemble de leurs membres.

Plus la taille du village est réduite et plus l'âge d'incorporation au cercle des vieillards tend à s'abaisser, afin de maintenir un effectif suffisant. À Konsogou-donyou, cet effectif était, en 1995, de 7 vieillards âgés de 66 à 87 ans, pour une population d'environ 225 habitants. Ce nombre était passé à 10 en 1997 et restait inchangé trois ans plus tard en l'absence de décès et de nouveaux entrants. Cependant, l'âge et l'existence de places vacantes ne sont pas les seuls critères déterminants: tous les chefs de lignage et de segments de lignage sont membres de jure de l'assemblée des Anciens, en tant que doyens de leur communauté respective. La "vieillesse » est donc une catégorie fondée sur la séniorité - faire partie des Aînés du village ou des têtes de lignage - plutôt que sur l'âge réel et le strict rapport d'aînesse: le doyen d'un segment de lignage, même s'il n'a que 65 ans, fait partie de plein droit des Anciens, contrairement à un homme d'un autre lignage pourtant plus âgé de quelques années. Ce double critère d'ancienneté, les Dogon l'expriment à travers certaines subtilités de langage. Deux hommes de Konsogou-donyou qui avaient rejoint le cercle des Vieux de manière anticipée, entre 67 et 69 ans, expliquaient par exemple: "Nous ne sommes pas encore complètement des nan garaa, mais nous sommes comptés parmi les nan garaa [les Anciens] $»^{12}$.

S'ils ont atteint tous les deux l'âge requis, un père et son fils peuvent-ils appartenir au même grade senior, en dépit de la règle d'évitement générationnel ? La faible espérance de vie et le recrutement tardif des Anciens rendent improbable une telle situation, jugée par ailleurs anormale. Ultime génération avant la mort et l'ancestralisation, les Anciens font le 
lien entre les vivants et les ancêtres wagi ("ceux qui sont au loin »), et c'est à ce titre qu'ils prennent en charge les rituels communautaires, villageois ou lignagers. Or, dans cette société où la filiation est patrilinéaire, seul un vieillard orphelin de père peut tenir ce rôle d'intermédiaire direct entre ses parents d'ici-bas et "ceux qui sont au loin ". Voilà pourquoi un homme dont le père est toujours en vie n'est pas autorisé à boire la bière offerte en libation aux ancêtres; ce qui l'exclut de fait du groupe des Anciens ${ }^{13}$.

Appartenant à la même génération - la troisième -, les Seniors font figure de "grands-pères " (baba); ils se comportent comme tels vis-à-vis à la fois de leurs propres petits fils (tiri-we) et du grade d'âge des sagators. Avec les premiers, ils entretiennent des relations affectives privilégiées, surtout si ces enfants sont très jeunes. Ils les emmènent par exemple au cabaret pour partager avec eux leur calebasse de bière, ou ils leur tiennent compagnie en jouant les baby-sitter attentionnés, contrairement aux "hommes» d'âge mûr qui agissent rarement ainsi, même si beaucoup d'entre eux ont déjà des petits-enfants. Seuls les Anciens se conduisent ouvertement comme des aïeuls débonnaires et tendres parce que leur statut et leur relative inactivité leur permettent justement de choyer leurs petits-enfants, alors qu'un "homme» ne peut se départir totalement de son rôle de chef de famille autoritaire, même vis-à-vis de ses petits-fils. En d'autres termes, un Dogon ne peut véritablement agir en grand-père avec ses petits-enfants que si son degré d'ascendance s'accorde avec son statut d'Ancien. Ajoutons que cette complicité entre générations alternées s'oppose à la froide indifférence entre un homme et ses arrière-petits-fils (tiri-we-timbe), ou à la relation d'évitement entre un homme et son arrière-arrière-petit-fils (sugu-tawale) ${ }^{14}$. De son vivant, chaque individu est enserré dans un réseau de relations qui ne va pas au-delà de deux générations ascendantes et descendantes.

Entre les vivants et les ancêtres, les relations se prolongent toutefois sur quatre générations ascendantes et descendantes, même si elles se concentrent sur les deux premières. Un ancêtre agnatique transmet une partie des composantes de sa personne à un ou plusieurs nouveau(x)-né(s) qui deviennent alors ses «répondants» (ncri). Cette transmission peut s'effectuer en ligne directe ou collatérale, sur quatre générations, mais l'ancêtre du répondant est presque toujours un frère aîné du père, un grand-père ou un frère du grand-père, plus rarement un arrière-arrière-grand-père, et en aucun cas

13. Les Anciens construisent une relation de continuité avec les ancêtres en versant régulièrement dans les poteries de ces derniers de la bière non fermentée, puis en la buvant un ou deux jours après (Jolly 1995 : 531).

14. Sugu-tawale signifie " [celui dont on] ne touche pas les oreilles". Il est interdit à un homme de toucher les oreilles de son arrière-arrière-petit-fils sous peine de le faire mourir, si bien qu'il s'abstient de tenir l'enfant dans ses bras ou même de s'en approcher. 
un père, un frère cadet du père ou un arrière-grand-père ${ }^{15}$. En ligne directe, les relations privilégiées entre générations alternées se manifestent donc par les liens unissant un neri à son grand-père défunt ou au grand père de ce dernier, tandis que l'absence de tout phénomène de "réincarnation ", entre un individu et son père décédé ou son arrière-grand-père, traduit une relation d'évitement entre générations adjacentes. Apparaît ici, en filigrane, un troisième système de "filiation " et de transmission patrilinéaire, plus personnalisé mais exprimant toujours de façon redondante l'auto-engendrement des hommes, que celui-ci s'effectue de père en fils, d'ancêtres en répondants ou, à l'échelle villageoise, de la classe des Hommes à celle des Jeunes.

Revenons justement à ces générations villageoises construites en faisant coïncider grade d'âge adulte et intervalle générationnel. Vis-à-vis des jeunes sagator de l'association de travail, les Vieux jouent à la fois sur le respect qu'ils inspirent et sur les traditionnelles relations de familiarité entre "grands-pères » et "petits-fils ». Ils viennent en groupe saluer les Jeunes lorsque ces derniers ont préparé ou obtenu de la bière, à l'occasion d'une fête ou d'un travail collectif. Les Vieux reçoivent alors une part de boisson et s'asseyent au milieu des Jeunes pour boire, avant de remercier et de repartir ensemble. En revanche, aucun "homme " d'âge mûr, de la génération des "pères", n'oserait entreprendre une telle démarche. Venir quémander de la bière chez ses " fils" serait en effet un acte parfaitement inconvenant.

Par opposition aux conduites et aux pouvoirs fortement individualisés des « hommes accomplis », les Vieillards agissent de concert, non seulement en tant que communauté de buveurs mais aussi en tant que dépositaires et gestionnaires des richesses lignagères ou villageoises. Les Jeunes, les Hommes et les Vieux occupent ainsi, vis-à-vis de la circulation du grain et de la bière, des positions complémentaires. Au sein de leur association de travail ou de leur groupe de parenté, les Jeunes cultivent pour les deux générations supérieures, tout en partageant entre eux la bière produite ou reçue. Les Hommes contrôlent la circulation du grain à l'intérieur de leur "famille» en bénéficiant des prestations de travail de leurs dépendants - fils et épouses - mais ils doivent aussi, vis-à-vis du reste du village, offrir du mil transformé en bière cérémonielle. Enfin, les Anciens contrôlent l'attribution des terres du lignage et la redistribution sous forme de bière des céréales rassemblées grâce aux contributions des chefs de famille. Bien entendu, ces positions ne sont jamais définitives: à l'échelle d'une vie, chaque individu passe successivement par ces trois échelons.

Le rôle économique et rituel des Vieux, aussi important soit-il, reste indépendant du pouvoir politique. Dans le contexte tøne, et contrairement à

15. Ces règles tene de désignation d'un neri semblent identiques, à quelques nuances près, à celles relevées par Germaine Dieterlen pour la région toro (1941: 128). 
d'autres régions voisines, il n'existe pas de véritable «conseil des anciens » délibérant pour administrer le village et juger les affaires en cours. D'ailleurs, les hommes les plus âgés du village n’ont jamais siégé au côté du ogo-waju, seul détenteur du pouvoir politique ${ }^{16}$. Ce chef sacré fondait son pouvoir exécutif sur plusieurs délégués - les ogว-seru et ogə-paru - nommés dans chacun des villages relevant de son autorité ou choisis à l'intérieur des lignages politiquement dominants. Ces représentants infligeaient des amendes aux auteurs de coups et blessures, aux voleurs et autres fauteurs de troubles, ou ils confisquaient les biens des meurtriers et des sorciers après les avoir traduits en justice devant le ogo-waju (Jolly \& Guindo 2003 : 16-22).

Dans les zones limitrophes, de tels pouvoirs policiers et judiciaires sont détenus par la société des masques, rejetée ici hors de la sphère politique et réduite à une institution relativement marginale, sans lien ou presque avec l'initiation masculine. En effet, les chefferies tøne semblent s'être développées au détriment de la société des masques, en récupérant certaines de ses prérogatives mais aussi en confisquant à son profit le prestige des masques en bois, dont la sortie est limitée à la seule levée de deuil du ogo-waju. Et à partir des années 1930, la disparition de ces chefs sacrés a précipité la fin des masques en bois tene en supprimant la seule occasion pour laquelle ils étaient fabriqués. L'organisation d'âge n'a pas davantage servi de socle ou de modèle au système politique tene. Nommé indépendamment du principe d'aînesse, le chef sacré échappait à tout classement dès son investiture ; il se situait au-dessus de la parenté, des générations et des catégories d'âge. En outre, il n'avait aucune prise ni aucun lien direct avec les classes d'âge, l'association de travail ou les vieillards en charge des rituels villageois; ce qui représentait d'ailleurs un frein à sa propre influence.

\section{Classes d'âge dono et Société des masques}

La situation est quasiment inverse chez les voisins occidentaux des T\&ne, dans les sous-régions dono de Taba-nonu et du Kamma ${ }^{17}$. En l'absence de pouvoir politique extérieur aux groupes de parenté ou aux Aînés, la Société des masques est, en pays dono, une institution centrale qui structure les classes d'âge masculines. Ces dernières sont en effet découpées au moment des rites d'incorporation à la Société des masques, entre 21 et 26 ans, c'est-à-dire bien après la circoncision, contrairement à l'exemple tहne.

16. Malgré tout, quelques villages tøne avaient à leur tête un chef sacré appelé garaa-ogo, investi à cette fonction en tant que doyen de sa localité. Ses sept suivants en âge - les ogo-kaana - l'assistaient et formaient avec lui un véritable "conseil des Anciens". La séniorité, dans ce cas, était le principe marquant de cette formule politique.

17. Les informations sur les classes d'âge dono proviennent essentiellement des travaux de Marcel Kervran sur le village de Pélou au Kamma (1999 : 98-103). 
Le principe de recrutement est le suivant : à l'intérieur du village, les vieux regroupent une grande partie des jeunes qui ont déjà approché les masques ajakay en agrégeant dans une même classe, et de façon définitive, tous ceux qui se suivent sur un intervalle de six ans. Cela correspond grosso modo à trois promotions de circoncis, mais des réajustements seront nécessaires pour écarter les plus jeunes et ne jamais dépasser ce double écart intergénésique au-delà duquel s'instaure une relation d'aînesse.

Simultanément, lors du rituel appelé "cotisations pour le Grand Masque », un des chefs de lignage devient le "grand-père » ( $b a b a)$ de cette classe d'âge des tumo-ulun. À l'échelle de la localité, chaque doyen de lignage est ainsi, à tour de rôle, le "grand-père paternel » d'une nouvelle promotion d'initiés; ce qui est une façon originale de concilier les principes lignagers et villageois (même si le village apparaît avant tout, dans cette configuration, comme une combinaison de lignages). Les membres nouvellement incorporés, organisés en groupe de travail, nomment en leur sein plusieurs responsables chargés de l'organisation des travaux collectifs ou de la discipline interne: un tums-amiri, chef de la classe d'âge, un tums-algal, faisant office de juge, un $t \varepsilon m$, responsable et porte-parole du groupe, un sonneur de trompe appelant au travail (kulu-suzi-ne), etc. (Kervran 1999: 102). Empruntée en partie aux sociétés voisines, cette titulature ne doit pas faire illusion: ces titres ronflants sont en fait partagés entre tous les membres du groupe en fonction des qualités qui leur sont reconnues. L'attribution de ces responsabilités temporaires ne s'oppose donc ni au principe d'aînesse qui régit la société dono, ni au principe d'égalité qui s'applique à toute classe d'âge.

Avant la formation de la classe d'âge suivante, les "nouveaux entrants" devront encore franchir deux étapes importantes au cours de deux levées de deuil successives. Ils vont d'abord "ramper autour du Grand Masque " (dannu yagule), nus et à quatre pattes, en se faisant flageller par les masques ajakay portés par leurs aînés. Après cette épreuve nocturne, point culminant de leur initiation, ils revêtiront pour la première fois le masque-cagoule "jeune fille» (i-nya) lors de la levée de deuil suivante. Mais les jeunes n'ont véritablement achevé leur initiation qu'à l'échelon supérieur, après avoir infligé à leurs cadets la fustigation qu'eux-mêmes ont subie. Ils peuvent dès lors tailler les masques en bois de leur choix.

En région dono, il existe un autre mode de classement de la population masculine, articulé lui aussi à la Société des masques et à l'initiation, à travers le célèbre rituel soixantenaire du sigi. Une nouvelle génération d' "enfants du sigi» (sigi-ulun) est engendrée tous les soixante ans par la promotion précédente des "vieux du sigi» (sigi pay). À cette occasion, tous les hommes qui n'ont pas encore été incorporés fournissent différentes 
prestations en grain pour "acheter" le sigi au vieux du village; et, au moment du rituel, ces novices de 1 à 59 ans défilent et boivent par ordre d'âge décroissant, sous le "patronage " du Grand Masque ${ }^{18}$. Célébrant la fécondité des hommes, cette cérémonie met ainsi en scène le renouvellement des générations masculines sous l'autorité exclusive des Vieux et des masques. Il n'est donc pas étonnant que le sigi soit présent en pays dono alors qu'il est absent dans la région voisine tene, où le pouvoir politique est indépendant des masques et du critère de séniorité.

Si les classes d'âge dono ont une assise villageoise, elles s'inscrivent finalement dans un ordre lignager où seuls les plus âgés détiennent l'autorité. Les membres des classes juvéniles sont explicitement à leur service : ils ont pour "grands-pères" les doyens de lignage et, sous couvert des masques ou de l'association de travail, ces Jeunes sont utilisés par les Vieux comme main d'œuvre agricole et comme force de police. En tant que danseurs masqués, ils «travaillent » également pour les vieillards défunts en participant à leurs levées de deuil et en contribuant ainsi à leur ancestralisation. Ces cérémonies funéraires sont d'ailleurs autant d'étapes obligatoires pour être admis dans la Société des masques, si bien que les nouveaux " entrants " doivent d'abord participer à l'ancestralisation des "partants ».

\section{Variante de Nombori}

\section{Des classes d'âge définies numériquement}

Gros village d'environ mille habitants, Nombori se trouve à l'intersection de trois zones linguistiques et culturelles: les régions tene au sud, dono à l'ouest et toro au nord-est. Cette situation particulière se traduit, au niveau de l'organisation sociopolitique, par une combinaison originale de traits empruntés à la fois aux Dono et aux T£ґe. Nombori est en effet la seule localité dogon où un chef sacré de type ogo-waju est associé à une Société des masques bien structurée. Le caractère insolite de cette association se répercute sur le système d'âge, tout aussi singulier : les classes d'âge, toujours au nombre de neuf, recrutent un nombre précis de membres et déterminent l'accès au groupe des Anciens.

Ce recrutement est précédé d'un accès progressif aux différents modèles de masques. Après leur incorporation à la Société enfantine des "Hyènes ", les jeunes garçons de 10 à 15 ans portent successivement, par groupe d'âge, trois ébauches de masque associés à un nombre équivalent d'échelons. Ces «masques enfantins" (uni lasugo) ne sont encore, à ce stade, que des

18. Ce Grand Masque dannu est un mât décoré, planté et dressé sur une place située un peu à l'extérieur du village. À la fois symbole phallique et représentation d'un ancêtre, il concourt à réaffirmer le rôle exclusif des hommes dans le processus de reproduction des promotions et des générations masculines. 
esquisses ou des ornements facilement dissociables du corps des garçons : un trait d'argile sur le ventre, un bandeau de fibres végétales puis un bouquet d'herbes au milieu du visage. À l'étape suivante, ces « initiés en herbe " revêtent des masques en feuilles de plus en plus volumineux et élaborés.

Les garçons, âgés au minimum de 22 ans, sont ensuite convoqués par les Vieux pour former une nouvelle classe d'âge et accéder simultanément à la Société des masques en fibres ou en bois. Ces jeunes s'alignent sur la place du village, par ordre d'âge décroissant, et les Aînés les comptent pour fermer leur promotion dès qu'ils arrivent à un nombre pair compris entre 40 et 46. Ce mode original de recrutement impose donc un découpage par dénombrement et non par intervalle de naissance, même si cette formule respecte l'espacement maximum de six ans propre à toute classe d'âge dogon. Les jeunes nouvellement recrutés se chargent de porter les masques en bois ou en fibres à l'occasion des levées de deuil ; ils participent également au rituel bisannuel du muns buro, en rejoignant à cette occasion les huit promotions précédentes. En prévision de cette cérémonie, chacune des neuf classes d'âge prépare de la bière en demandant à ses membres de verser exactement la même quantité de mil. Une petite partie de la boisson ainsi brassée est apportée sur le lieu du rituel pour être transvasée dans une grande jarre réservée à l'usage exclusif des Anciens. À tour de rôle et par ordre d'ancienneté, les classes d'âge se présentent devant l'autel muns. Leurs membres sont rangés en file indienne, du plus âgé au plus jeune, et tous s'accroupissent au moment où le sacrificateur égorge leur poulet, puis ils partent consommer le restant de leur bière chez celui qui l'a brassée. Quant aux Anciens, ils demeurent sur place pour boire une grande partie de la nuit.

Comme il n'existe que neuf classes d'âge, regroupant tous les hommes âgés de 24 à 69 ans environ, la formation d'une nouvelle promotion pousse automatiquement celle qui occupait le neuvième rang dans le groupe des muns bors deฑe, "ceux qui sont assis sous l'autel muns " ${ }^{19}$. Ce titre, attribué aux Anciens du village, se réfere explicitement à leur station assise durant le rituel précédemment esquissé, par opposition aux autres participants «accroupis ${ }^{20}$, mais l'expression évoque plus généralement leur position d'Aînés, responsables du village. Comprenant une trentaine de Vieux en moyenne, le groupe des muns bors deฑE, possède des prérogatives rituelles, une autorité morale et un rôle de conseillers auprès des doyens de lignage et des deux chefs sacrés de Nombori. Bien entendu, ces pou-

19. L'autel muns du quartier Gomu-na de Nombori se trouve dans la fourche d'un tamarinier, si bien que les participants se situent sous cet autel.

20. Les membres des neuf classes d'âge sont d'ailleurs désignés par l'expression mun b bro tonren, «ceux qui s'accroupissent sous l'autel muns". 
voirs ne se transmettent pas : si les hommes accèdent au statut d'Anciens par le biais du système de classes d'âge, ils s'y maintiennent ensuite jusqu'à leur mort, au nom du principe de séniorité.

\section{Le modèle "fédéral" tomว}

\section{Des classes d'âge régionales}

La région tomว se subdivisait autrefois en "fédérations villageoises" regroupant chacune une douzaine de localités non apparentées ${ }^{21}$. Entre village et chefferie, cette formation politique intermédiaire s'inspirait des kafo bamana et remontait sans doute à la domination du royaume de Ségou sur toute cette partie méridionale du pays dogon. Chacune de ces "chefferies" épousait les contours d'un territoire de chasse et correspondait initialement à un ensemble politique et militaire doublé d'un espace matrimonial, judiciaire et commercial. Les charges politiques et rituelles (chef de terre, chef de guerre, chef des masques, maître de la chasse, chef sacré, responsable du marché, etc.) étaient réparties entre les villages en étant, pour certaines, attribuées à tour de rôle à chacune de ces localités, indépendamment des relations de parenté. Les classes d'âge, telles qu'elles existaient au début de ce siècle, s'inséraient dans ce dispositif régional, mais le démantèlement des fédérations après la colonisation française, puis la conversion des jeunes à l'islam, ont profondément modifié les institutions tomว. Les informations qui suivent ne portent donc pas sur la situation actuelle mais sur celle qui prévalait jusqu'au premier tiers de ce siècle dans la fédération de Arou-na, sur le massif de Garou, et dans le village voisin de Ama, issu des douze «portes» de l'agglomération de Toumou ${ }^{22}$.

Pour les garçons âgés environ de quinze ans, la circoncision avait lieu la même année, à un jour d'intervalle, dans tous les villages de la fédération. Les nouveaux circoncis entraient d'abord dans l'association de culture de leur quartier et ils y restaient une douzaine d'année, travaillant à la demande et contre paiement. Vers l'âge de 26 ans, et cette fois à l'échelle de toute la fédération, les jeunes accédaient ensemble à l'échelon des tsbie uniwe, en se plaçant simultanément sous les ordres d'un maître de la guerre, «champion de brousse » (bala seri). Corvéables à volonté et au service de leur fédération, les tobie uniwe - «enfants qui frappent (?)»étaient, suivant les circonstances, guerriers, bûcherons, ouvriers agricoles ou fossoyeurs. À l'échelon suivant, deux ans plus tard, ils devenaient des "enfants des masques", lesuba uniwe. Ils confectionnaient leurs masques

21. Du reste, les habitants de chacun de ces villages portent un "nom de famille" spécifique.

22. Le modèle originel de ces fédérations aujourd'hui éclatées est en effet une grosse "agglomération " constituée de douze «quartiers » bien distincts, sans liens de parenté. 
en brousse puis les revêtaient et convergeaient le dixième jour vers le centre du territoire. Classés par village, du plus ancien au plus récent, ils formaient une même procession linéaire et, sous la conduite du chef des masques (paran), ils effectuaient le tour de leur fédération en s'agenouillant devant l'abri des hommes de chaque localité. Les "enfants des masques » assuraient ensuite la "police » pendant deux ans, à l'intérieur de la fédération, et ils accédaient enfin à un dernier échelon appelé parfois on o ou one, du nom du masque "singe rouge" qu'ils portaient pour patrouiller dans les villages.

Si l'association de travail peut être considérée comme un simple complément à l'organisation lignagère, les classes d'âge occupant les trois échelons suivants étaient donc étroitement associées au processus initiatique, à la sphère politique et à l'organisation territoriale ; elles étaient d'ailleurs affectées à la défense de cet espace régional, soit sous forme de détachement militaire, soit en tant que "force de l'ordre ». Mais l'organisation politique dont dépendaient ces échelons initiatiques avait peu de points communs avec une chefferie classique, où le pouvoir est détenu par un seul homme et hérité à l'intérieur d'un même lignage. Dans la configuration tomว, où le pouvoir est au contraire partagé, alterné et limité dans le temps, les classes régionales de guerriers et de " policiers » étaient dirigées respectivement par un maître de la guerre et un chef des masques qui changeaient tous les deux ans, en étant choisis dans les différents villages de la fédération.

Certains «champions de brousse " réussissaient toutefois à imposer leur leadership à travers un défi et une épreuve magico-guerrière où ils démontraient publiquement leur supériorité (Jolly 1998-99). On peut donc se demander si un chef de guerre tomว particulièrement ambitieux ne pouvait pas s'appuyer sur la classe des «enfants qui frappent " pour confisquer et monopoliser le pouvoir, à l'image d'un Biton Coulibali, qui avait fondé le royaume bamana de Ségou, au XVIII ${ }^{\mathrm{e}}$ siècle, en se servant des ton-den, membres d'une association d'âge, agricole ou guerrière. Mais l'État ainsi créé ne vivait que de la guerre et des tributs versés par les régions conquises, et les ton-den ont été rapidement supplantés par une armée de ton-jon, guerriers de condition, d'ethnies et d'âges très divers, au recrutement hétéroclite et au service exclusif du roi. Or, dans le contexte toms, les "enfants qui frappent" n'étaient comparables ni aux ton-den ni aux ton-jon. Certes, ils s'apparentaient à une armée de travailleurs et de combattants disciplinés, mobilisables à volonté, mais ils correspondaient à une classe éphémère de "novices " au service de leur communauté et non à une association susceptible de se placer durablement sous les ordres d'un seul homme. En outre, ils étaient uniquement affectés à la défense d'un territoire et n'avaient pas pour vocation de participer à des razzias ou à des 
guerres de conquête. De fait, aucun bala seri n'a jamais manipulé ou contrôlé à son profit la classe des tsbie uniwe, en l'attirant dans ses aventures guerrières. Si certains chefs de guerre toms étaient devenus menaçants, c'est uniquement parce qu'ils avaient entrainé derrière eux un petit groupe de partisans et de guerriers semi-professionnels - les bata - dont l'engagement individuel et volontaire était incompatible avec la logique communautaire des classes d'âge.

\section{Les classes d'âge mixtes de Koundou (région toro)}

Le dernier modèle examiné se singularise par la mixité et la formation tardive de ses classes d'âge. Koundou, en zone toro, est un ensemble de cinq villages voisins et apparentés, dont les habitants portent tous le même «nom de famille » : Dara. Contrairement au cas tomı analysé précédemment, l'élément fédérateur est ici la parenté, réelle ou fictive : tous les villages de l'agglomération se réclament d'un ancêtre-fondateur commun, et leur chef sacré - le lebe-sgonu - est toujours le doyen de tous les Dara de Koundou et des localités qui en sont issues. Ce type d'organisation supra-villageoise, fondé sur la parenté et la séniorité, n'a donc aucun rapport ni avec une chefferie classique (où le pouvoir se transmet de manière héréditaire) ni avec un système fédéral (où le pouvoir est morcelé, partagé ou alterné).

À Koundou, les garçons sont circoncis vers quatorze ans, par village, et leur promotion (tono) s'agrège ensuite à l'association de culture de leur localité, ou aujourd'hui de leurs quartiers. Quant aux filles, jamais excisées, elles se constituent en groupe de travail parallèlement à la promotion de circoncis correspondant à leur tranche d'âge. Une fois sortis de l'association de travail, vers quarante ans, les individus nés à Koundou dans un intervalle de deux ans étaient regroupés dans une même "classe d'âge" (kadaga). J'utilise volontairement l'imparfait parce que la formation du dernier kadaga de Koundou remonte au début des années $1960^{23}$. Le découpage de cette classe d'âge était public: les Anciens nommaient par ordre d'âge décroissant tous les membres du kadaga, hommes et femmes, en s'arrangeant pour que l'aîné de la promotion soit toujours de sexe masculin $^{24}$. Les individus ainsi "appelés » étaient aspergés de bière puis regagnaient leur foyer, parfois dans des villages éloignés dans le cas des femmes de Koundou mariées à l'extérieur.

23. Aujourd'hui, un homme de Koundou peut dire, de façon plus ou moins impropre, que tel individu est du même kadaga que lui, mais cela signifie simplement qu'ils appartiennent au même intervalle d'âge de deux ou trois ans.

24. Pour donner un ordre de grandeur, le dernier kadaga de Koundou comptait une soixantaine de personnes des deux sexes pour une agglomération dont la population dépassait sans doute, en 1960, les 1100 habitants actuels. 
Deux mois plus tard, ils "entraient dans le kadaga" en s'enfermant ensemble dans la concession de l'aîné. Ils y restaient cloîtrés durant cinq jours, avec interdiction pour eux d'en sortir mais aussi pour les autres d'y entrer. Du sixième au dixième jour, les membres du kadaga commençaient à boire ensemble la bière préparée avec le mil qu'ils avaient cotisé, en excluant de cette consommation commune toute personne extérieure à leur groupe. Au cours de cette seconde période, ils défilaient également dans l'agglomération, parés de leurs plus beaux habits et rangés dans une même file par ordre d'âge décroissant, sans distinction de sexe. Enfin, le dixième jour, les membres du kadaga se séparaient pour la première fois en deux colonnes parallèles, en fonction de leur sexe. Hommes et femmes étaient alors appariés pour former des couples d'amis (anige): l'aîné des hommes devenait l'ami de l'aînée des femmes, et ainsi de suite jusqu'au benjamin. Si le groupe comptait davantage de membres féminins que masculins, le plus âgé reprenait une seconde amie, son suivant aussi, etc. À cette occasion, chaque homme offrait cinq cauris à sa camarade qui, en retour, offrait ultérieurement un canari de bière à son "alter ego » de l'autre sexe, afin de sceller leur amitié institutionnelle, strictement platonique.

Cette classe d'âge a de quoi surprendre: elle est formée d'individus d'âge mûr, d'une quarantaine d'années; elle rassemble à la fois des hommes et des femmes en les plaçant sur un plan d'égalité; et elle se constitue sans jamais s'articuler avec un processus initiatique. En outre, on peut s'interroger sur l'utilité d'un tel kadaga, en l'absence de toute activité spécifique. Et pour achever de nous dérouter, l'amitié entre un homme et une femme prend la forme d'une liaison imposée au lieu d'être une relation élective. Pourtant, le sens d'une telle classe d'âge s'éclaire en utilisant le modèle interprétatif appliqué à l'exemple tene. La constitution d'un kadaga n'est compréhensible en effet que dans une perspective "générationnelle». Les camarades d'âge des deux sexes se regroupent vers 40-45 ans non pas pour se positionner par rapport à leurs cadets ou leurs aînés immédiats, absents de l'ensemble des rites, mais pour opérer leur passage à la "génération" supérieure, juste après leur sortie de l'association de culture (réunissant une même génération de "fils»).

$\mathrm{Ni}$ main d'œuvre agricole ni force de police, les membres d'un kadaga étaient, au sens propre comme au figuré, des pères et des mères de famille définitivement "rangés » et "reclassés ", ne travaillant plus pour la collectivité. Cela apparaît clairement dans les rites décrits précédemment: les nouveaux entrants ne se mettent au service de personne; ils se coupent au contraire du reste de leur communauté en s'enfermant dans une concession pour vivre et boire ensemble de manière solidaire et égalitaire. Même la formation de couples d'amis s'inscrit dans cette logique générationnelle. 
À Koundou comme dans les autres régions dogon, la consécration d'une amitié platonique entre un homme et une femme a toujours lieu après quarante ans, lorsque les deux partenaires d'âge mûr ont, chacun de leur côté, une relation conjugale stable qui lève toute ambiguïté sur leurs propres rapports (Jolly 1995: 451). En retour, l'institutionnalisation de leur amitié est une reconnaissance publique de leur statut de conjoints et de parents accomplis, définitivement assagis, par opposition justement à la vie sexuelle aventureuse ou réputée telle des jeunes de la génération suivante, qu’ils soient célibataires ou jeunes mariés. À Koundou, la répartition de tous les membres du kadaga en paires d'amis de sexe opposé prend toutefois une forme systématique qui n'a pas d'équivalent dans les autres régions; mais cet appariement en fonction de l'âge repose finalement sur une règle implicite valable pour l'ensemble du pays dogon : l'ami(e), que l'on traite en égal(e), ne peut être, idéalement, qu'un(e) camarade de même âge. Et selon cette même logique poussée à l'extrême, deux personnes de sexe opposé et ayant exactement le même rang d'âge sont forcément faites pour aller ensemble, non pas en tant que mari et femme mais en tant que couple d'amis conjoints. Du reste, dans de nombreux parlers dogon, les termes désignant le camarade d'âge, le compagnon ou l'ami sont soit identiques, soit plus ou moins synonymes et intervertibles, par le jeu des euphémismes.

Il reste à comprendre pourquoi les classes d'âge de Koundou, et elles seules, autorisaient la mixité et se construisaient à la génération des "pères ». L'explication réside sans doute dans l'absence de toute connexion entre classes d'âge et échelons initiatiques. À Koundou, ce ne sont pas les jeunes qui subissent une initiation ou qui " payent " pour avoir accès aux masques, ce sont au contraire les hommes d'âge mûr qui, individuellement et librement, offrent une certaine quantité de bière pour avoir droit à une sortie de masques lors de leurs propres funérailles.

Un tel exemple ne doit pas faire illusion. L'organisation d'âge dogon, à dominante masculine, n'accorde aux femmes qu'une place relativement marginale et subalterne. À Koundou, elles sont certes classées en fonction de leur âge mais parallèlement aux hommes et en leur abandonnant la place d'aîné. Cependant, si les deux sexes sont regroupés et appariés, ce n'est pas tant pour souligner le statut d'éternelle cadette de la femme que pour manifester leur appartenance originelle à une même agglomération. Cette mixité permet en effet de réaffirmer la primauté de la filiation sur l'alliance à travers la réunion de tous les "fils " et "filles " de Koundou engendrés dans le même intervalle et séparés depuis par le mariage. Dans les autres régions dogon, les groupes d'âge féminins, quand ils existent, sont peu marqués et toujours en retrait par rapport aux classes masculines 
correspondantes. Pour les femmes dogon, la division essentielle repose moins sur l'âge que sur la distinction entre « sœurs » natives du village et " étrangères " mariées dans cette localité (cf. Annexe).

\section{La force de l'“âge" dans la société dogon}

Dans cette société patrilinéaire et patrilocale, si la distribution des femmes obéit avant tout à une logique d'alliance, le classement des hommes reproduit en revanche le modèle de la filiation agnatique. Les hommes du village, tous lignages confondus, se rangent en classes et en échelons d'âge successifs en s'inscrivant dans une « filiation » en ligne masculine où chaque classe et chaque "génération " engendrent fictivement la suivante. Et leurs charges politiques, rituelles et économiques se distribuent en grande partie en fonction de cette stratification.

\section{Générations et économie}

Rapports de production, répartition des ressources et circulation des richesses sont eux-mêmes régis par la division de la société villageoise en trois générations adultes: Anciens, Hommes et Jeunes. Prendre en compte ces catégories générationnelles permet de dépasser la traditionnelle opposition entre aînés et cadets mise en avant par le courant anthropologique d'inspiration marxiste. Initiée à partir des années 1960 puis critiquée par Marc Abélès et Chantal Collard (1985 : 10-11), l'analyse fondée sur les rapports de domination des aînés sur les cadets s'est révélée en effet insuffisante pour éclairer la complexité des échanges et des rapports de production, d'autant qu'un individu est toujours le cadet et l'aîné de quelqu'un. En pays dogon, il n'y a pas, d'un côté, une classe des cadets qui travaille et produit pour les aînés, sous leur autorité, et, de l'autre, une classe des aînés qui se charge de la redistribution des produits. Libérés de l'association de culture, les "Hommes", par exemple, ne travaillent plus pour la collectivité mais, en tant que chefs de famille, ils contribuent aux dépenses rituelles en versant leur quote-part aux Anciens. Les rapports de production ne sont donc pas réductibles à une relation duelle; et l'intérêt de cette étude est justement de révéler l'importance du critère générationnel pour toute analyse de l'organisation socio-économique ${ }^{25}$.

\section{Diversité des formules dogon}

Autre avantage d'une telle recherche: elle dévoile la surprenante variété des dispositifs de classement. Certains s'additionnent, d'autres se déploient duction développée dans ma thèse sur la bière de mil dans la société dogon (1995). 
dans des régions ou des localités différentes, et toutes évoluent dans le temps; mais cette diversité va de pair avec l'existence de classes d'âge peu affirmées, voire à peine amorcées. Les différentes formules examinées ne correspondent jamais à des "système de classes d'âge " modelant l'ensemble de l'organisation sociopolitique. À propos des Dogon, les ethnologues parleront tout juste de "société à classes d'âge ", en accompagnant cette définition de multiples réserves tant les classes d'âge dogon paraissent timides comparées à d'autres modèles plus classiques. L'erreur, cependant, serait de conclure un peu vite à la pauvreté ou à la banalité de l'organisation d'âge dogon. C'est justement l'absence de système ou de modèle déterminé pesant sur l'ensemble de la société qui autorise un large éventail de combinaisons, en permettant à chaque dispositif de s'adapter aux particularités et aux évolutions locales ou régionales.

Chaque village ou région dogon a su inventer une formule originale en privilégiant selon le contexte: la défense du territoire (en pays toms), l'unité intangible des " enfants » d'une même agglomération (à Koundou), l'association de travail (en zone tøne), les échelons initiatiques de la Société des masques (en région dono), l'effectif constant des danseurs masqués et des Anciens (à Nombori). Et ces exemples sont loin d'être exhaustifs : d'autres dispositifs existent ou ont existé à l'intérieur des frontières du pays dogon, par exemple au Wadouba (Holder 1999: 479).

\section{Âge et systèmes politiques}

Reposons maintenant le problème des rapports entre classes d'âge et formations politiques dogon. Les classes d'âge juvéniles les plus affirmées, ou du moins celles qui s'articulent avec un système d'échelons initiatiques, sont celles qu'un pouvoir politique de type collégial ou fédéral organise en force policière ou militaire. En région dono, les classes d'âge en formation correspondent ainsi à des promotions d'initiés incorporant par étapes la Société des masques et agissant pour le compte des vieux, au bénéfice également de l'ordre masculin et lignager. Quant aux classes d'âge juvéniles tomว, organisées en détachement guerrier puis policier, elles étaient l'instrument d'un pouvoir fédéral assuré non pas collectivement mais alternativement.

Or, la répartition des tâches entre classes d'âge ne se réalisait justement que dans le contexte toms, où le pouvoir était lui-même fractionné et distribué entre des villages ordonnés en fonction de leur ancienneté. La concordance de classement entre les hommes et leurs lieux de résidence était particulièrement flagrante à deux occasions. Les jeunes de la fédération de Arou-na étaient circoncis, rappelons-le, par village, à un jour d'intervalle, en commençant par la localité la plus ancienne et en finissant par la plus récente, afin que leurs promotions se conforment au même ordre 
d'antériorité. En outre, lors du défilé des nouveaux «enfants des masques ", tous les jeunes de cet échelon étaient incorporés dans une même file en étant d'abord classés par village, par ordre décroissant d'ancienneté, puis par âge, de l'aîné au benjamin. Et cette file effectuait le tour de la fédération en suivant l'ordre de fondation des douze localités. Ce type de classement, des hommes comme des lieux de résidence, n’obéissait à aucun principe hiérarchique et ne créait aucun rapport de subordination ; il garantissait au contraire le caractère unitaire et égalitaire de toute fédération et de toute classe d'âge toms. D'ailleurs, les fonctions de chef de guerre et de chef des masques passaient également de village en village. En imposant une rotation et une répartition des responsabilités militaires et judiciaires, les classes d'âge tom o interdisaient toute concentration des pouvoirs au profit d'un seul groupe ou d'un seul individu.

Inversement, dès qu'apparaît un embryon de chefferie, dès que le pouvoir est détenu durablement par un seul homme ou un seul lignage, les classes d'âge sont beaucoup plus simples et extérieures à la fois au processus initiatique et à la sphère politique. En région têje, elles se réduisent à de banales promotions de circoncis au découpage approximatif, tandis que les kadaga de Koundou étaient des "promotions d'âge " isolées et mixtes, totalement indépendantes de la société des masques et du pouvoir exécutif. Ces classes d'âge sont liées davantage aux "générations » qu'aux échelons initiatiques; elles permettent soit de construire la génération laborieuse des fils (par regroupement de toutes les classes juvéniles dans une même association de travail), soit de passer à la génération supérieure (grâce à la création d'une promotion d'hommes et de femmes parvenus à une pleine "maturité sociale»).

Les exemples dogon viennent donc confirmer l'hypothèse ou tout simplement le constat avancé par de nombreux ethnologues: les classes d'âge les plus structurées semblent incompatibles avec un pouvoir centralisé et durable (Paulme ed. 1971 : 11 ; Bernardi 1985: XIV ; Peatrik 1995: 8-9). En revanche, elles se combinent parfaitement avec un système politique privilégiant la défense ou le contrôle d'un territoire commun (Peatrik 1999 : 508), à l'instar des modèles tom et dono. Seul le cas de Nombori semble a priori contredire cette démonstration, avec la présence côte à côte de classes d'âge structurées et de chefs de type ogo-waju. Mais, à y regarder de plus près, la centralisation du pouvoir est finalement toute relative dans cet exemple : il existe en effet deux ogo-waju pour la même localité, et non un seul chef pour un groupe de villages. D'autre part, les classes d'âge de Nombori dépendent avant tout de l'autorité collégiale des Aînés, «assis sous le muno ".

Qu'en est-il du rapport plus controversé entre classe d'âge et lignage ? À la suite d'Einsenstadt (1956), de nombreux chercheurs ont opposé les deux 
institutions: les systèmes de classes d'âge ne se développeraient dans les sociétés villageoises qu'au détriment du lignage et de la parenté. Pour Denise Paulme, par exemple, l'importance des classes d'âge "se révèle inversement proportionnelle à celle des lignages » $(1971: 16)$. Stéphan Dugast aboutit à des conclusions similaires en reprenant l'analyse des sociétés lagunaires de Côte-d'Ivoire (1995 : 145, 154). À l'inverse, Jean Jamin conteste l'opposition entre les deux institutions en faisant remarquer que les classes d'âge s'accommodent très bien de l'ordre lignager et paraît même le conforter, en masquant son caractère inégalitaire (1977 : 65-66, 84).

Les données dogon peuvent-elles faire avancer ce vieux débat? Dans les cinq exemples retenus, les classes d'âge dogon endossent partout une dimension villageoise ou régionale tout en composant avec les lignages. L'intrication est telle, chez les Dono, qu'une nouvelle classe d'âge a toujours pour «tuteur » un chef de lignage, choisi par rotation au sein du village. Or cette formule dono ne concurrence pas le lignage, pas plus qu'elle ne travestit sa hiérarchie interne ; elle le complète plutôt, notamment par l'intermédiaire de la Société des masques (qui organise les classes masculines). Je rejoins par conséquent le point de vue exprimé par Anne-Marie Peatrik (1995: 8) : il ne faut plus penser les classes d'âge par rapport au lignage mais par rapport aux diverses formations politiques avec lesquelles elles s'articulent - formations de type collégial ou « fédéral » qui autorisent une structure aussi bien lignagère, villageoise que territoriale, avec toutes les combinaisons possibles.

\section{De la filiation au défilé des âges}

Une question reste en suspens : pourquoi la société dogon manifeste-t-elle une telle insistance à affirmer son mode de filiation patrilinéaire? De toute évidence, l'objectif est d'étendre ce mode de filiation au-delà de la sphère de parenté pour l'appliquer avec plus ou moins de force aux communautés locales ou territoriales: village, agglomération, fédération ou même portion du pays dogon (pour toute la zone traversée par le rituel soixantenaire du sigi). Pour imposer ce principe de filiation "résidentielle », l'un des procédés les plus spectaculaires est sans doute le déplacement des classes d'âge à l'intérieur de ces espaces villageois ou régionaux. De tels défilés dessinent ostensiblement l'image d'un groupe se succédant le long d'une chaîne continue, depuis les ancêtres masculins jusqu'aux nouveau-nés de même sexe.

Rangées en file indienne, par ordre d'âge décroissant, les classes d'âge assurent la cohésion de leur communauté en l'inscrivant à la fois dans une continuité temporelle et dans un espace commun. Elles lui donnent l'illusion de sa reproduction linéaire et, simultanément, elles relient ses diffé- 
rentes composantes en défilant dans l'ensemble de ses quartiers ou de ses localités. S'étirant d'un lieu à un autre, ces files de camarades d'âge mettent en effet en scène, d'une part, la succession des naissances ou le renouvellement des générations et, d'autre part, l'unité du groupe partageant le même espace et les mêmes institutions.

Dans le village dono de Pélou, les jeunes hommes qui constituent une nouvelle classe d'âge défilent soit par quartier, pour se fondre ensuite dans une colonne unique (Kervran 1999: 101), soit par village, en s'arrêtant pour chanter devant l'abri des hommes de chaque quartier (ibid. : 296). À Koundou, ce sont les hommes et les femmes d'un même kadaga qui se déplacent devant les principaux autels de leur agglomération en étant rangés, par ordre d'âge décroissant, dans une même file serpentine. Enfin, on vient de le voir, les jeunes lesuba-uniwe d'une fédération tomo, s'ils s'inséraient dans une seule file, restaient toutefois groupés par village pour défiler dans toutes les localités du territoire. Quant au long cortège des " enfants du sigi ", il forme tous les soixante ans une chaîne ininterrompue unissant non seulement les générations successives, mais aussi, de proche en proche, les différentes localités toro et dono qui se transmettent le rituel. Ces colonnes se rejoignent ou se séparent, se suivent ou se croisent, en étant découpées, selon les cas, par lignage, village ou groupe de villages. Voilà bien la preuve que le système de classes d'âge combine constamment ces trois formes d'organisation, sans exclusive, y compris au sein d'une même formation politique.

Seule la région tęe n'est pas "traversée " par ce type de défilés unissant successivement, dans le temps et l'espace, tous les membres d'une même communauté. Les nouveaux circoncis se contentent de parader dans le village sans vraiment respecter de parcours précis, tandis que le ogo wajudétenteur du pouvoir politique - ne prend la tête d'aucune colonne. Extérieur à la Société des masques et aux classes d'âge, ce chef est le seul à effectuer le tour de l'agglomération, lors de son investiture, afin d'inscrire son pouvoir sur ce territoire et garantir la cohésion de celui-ci (Jolly, 199899 ; Jolly \& Guindo 2003 : 27-28). Dans le contexte t£Đe, le périple solitaire du chef semble avoir interrompu la file des âges.

MOTS CLÉS/KEYWORDS : âge/age - Dogon - génération/generation - patrilinéarité/patrineal descent - anthropologie politique/political anthropology. 
Abélès, Marc \& Collard, Chantal, eds

1985 Âge, pouvoir et société en Afrique noire. Paris, Karthala/Montréal, Presses universitaires de Montréal.

\section{Bernardi, Bernardo}

1985 Age Class Systems. Social Institutions and Polities Base on Age. Cambridge,

Cambridge University Press.

Bouju, Jacky

1984 Graine de l'homme, enfant du mil.

Paris, Société d'ethnographie.

Calame-Griaule, Geneviève

1965 Ethnologie et langage. La parole chez les Dogon. Paris, Gallimard.

1968 Dictionnaire dogon (dialecte toro).

Langue et civilisation. Paris, G. Klincksieck.

Dieterlen, Germaine

1941 Les Âmes des Dogons. Paris, Institut d'ethnologie.

Dugast, Stéphan

1995 «Lignages, classes d'âge, village. À propos de quelques sociétés lagunaires de Côte d'Ivoire ", L'Homme 134 : 111-157.

Eisenstadt, S. N.

1956 From Generation to Generation: Age Groups and Social Structure. New York, The Free Press (rééd. 1971).

Héritier, Françoise

1981 L'Exercice de la parenté. Paris, Le Seuil.

Holder, Gilles

1999 Le Système politique sama. Parcours et relations d'une société guerrière dans la boucle $d u$ Niger: analyse comparative. Nanterre, Université Paris X, thèse de doctorat.

Jamin, Jean

1977 Les Lois du silence. Essai sur la fonction sociale du secret. Paris, Maspero.
Jolly, Éric

1995 La Bière de mil dans la société dogon.

Nanterre, Université Paris X,

thèse de doctorat.

1998-1999 "Chefs sacrés et chefs de guerre dogon : deux pôles du pouvoir ", Clio en Afrique 5 [http ://www.up. univmrs.fr/ $\sim$ wclio-af/].

\section{Jolly, Éric \& Nouhoum Guindo}

2003 Le Pouvoir en miettes : récits d'intronisation d'un hogon (pays dogon, Mali). Paris, Classiques africains.

\section{Kervran, Marcel}

1993 Dictionnaire dogon-français donno so. Région de Bandiagara. Bruxelles, R. Deleu.

1999 La Vie et la mort en pays dogon. Rites et célébrations chez les Donnon. Chez

l'Auteur.

\section{Paulme, Denise}

1940 Organisation sociale des Dogon. Paris, Domat-Montchestien (rééd. Paris, JeanMichel Place, 1988).

1968 " Pacte de sang, classes d'âge et caste en Afrique noire ", Archives européennes de Sociologie 9: 12-33.

1971 "Les classes d'âge dans le sud-est de la Côte-d'Ivoire ", in Denise Paulme, ed., Classes et association d'âge en Afrique de l'Ouest. Paris, Plon : 205-285.

Paulme, Denise, ed.

1971 Classes et association d'àge en Afrique de l'Ouest. Paris, Plon.

\section{Peatrik, Anne-Marie}

1995 "Introduction", L'Homme 134 : Âges et générations: 7-12.

1999 La Vie à pas contés: génération, âge et société dans les hautes terres du Kenya (Meru Tigania-Igembe). Nanterre, Société d'ethnologie. 


\section{L'âge au féminin}

\section{$\mathrm{L}$}

ES FEMMES tEทe : de la filiation à l'alliance. Pour la région tene, il serait tout à fait exagéré de parler de classes d'âge ou même de "générations " féminines. L'excision est pourtant l'équivalent féminin de la circoncision et, tous les deux ou trois ans, elle crée effectivement des promotions de fillettes de même âge, mais celles-ci n'ont pas les mêmes caractéristiques que les classes d'âge masculines. La précocité de l'excision par rapport à la circoncision est une des premières explications de ces différences ; l'opération, pratiquée entre 4 à 6 ans, n'entraîne ni passage à l'âge adulte ni changement de statut : la fillette excisée continue à vivre et à travailler chez ses parents, contrairement aux nouveaux circoncis. Les cérémonies et la réclusion qui entourent l'excision sont d'ailleurs très sommaires : 10 jours de retraite contre 35 pour les garçons, pas de chants à mémoriser et pas d'initiation proprement dite. Enfin, les fillettes ne sont pas surveillées par leurs aînées de la promotion précédente - bien trop jeunes - mais par les femmes les plus vieilles, natives du village, à l'exclusion des " étrangères ". Dans ce contexte, l'excision s'apparente moins à une opération de classement qu'à un rite d'incorporation villageoise.

À Konsogou-donyou, seuls quatre groupes d'excisées sont membres de l'association de culture ; et dès qu'une nouvelle promotion entre, vers 15 ans, la plus âgée en sort, sans jamais passer par des échelons intermédiaires. Toutefois, les jeunes filles quittent rarement l'association de travail en même temps; elles en sortent plutôt individuellement lorsqu'elles partent s'installer chez leur mari dans un autre village ou quartier, à partir de 21-22 ans. Les filles se raccrochent ainsi de manière marginale à une association de travail largement dominée par les hommes. Elles n'occupent que les quatre premiers échelons, jusqu'au premier tiers de ce groupe, et elles participent certes au battage du fonio mais sous autorité et encadrement masculins. Les deux "accompagnateurs de jeunes filles» taillent en effet leurs fléaux et les escortent lors de leurs déplacements nocturnes, tandis que le responsable masculin - le buru dine baya - exerce également son autorité sur les filles, en ayant le pouvoir de leur infliger des amendes.

Les filles astreintes aux travaux collectifs possèdent toutefois une certaine autonomie par rapport à leurs partenaires masculins, d'une part, parce que certaines 
tâches leur sont réservées, en particulier le transport du fumier dans les champs ${ }^{26}$, et d'autre part, parce quelles se choisissent tous les deux ans une "tutrice" selon deux critères : il doit s'agir d'une femme d'âge mûr et d'une étrangère mariée dans le village (ya yere). Prenant le titre de nàanaa, "grand-mère maternelle", cette femme est secondée par une adjointe, forcément plus jeune et mariée dans un autre lignage. La nàanaa ne se manifeste pas au moment des travaux collectifs; elle intervient uniquement au cours des festivités organisées par les jeunes filles en leur offrant de la bière et en les aidant à en préparer. La présence d'une "grand-mère" n'appartenant pas au grade d'âge des vieilles traduit le brouillage des degrés d'âge féminins. Il serait d'ailleurs vain de chercher des échelons correspondant à des "générations " de filles, de mères et de grands-mères; les femmes n'ont que des catégories d'âge aux contours relativement flous, contrairement aux hommes qui bénéficient de bornes précises comme la circoncision, la sortie de l'association de travail ou l'incorporation au "conseil des Anciens». D'autre part, à l'intérieur du groupe des femmes mariées, la principale division ne repose pas sur l'âge mais sur la distinction entre épouses natives du village et

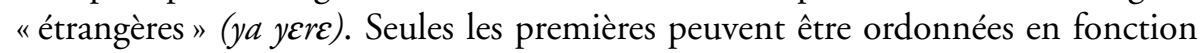
de leur âge, et la position de doyenne ou d'aïeule leur est d'ailleurs réservée.

\section{Classes d'âge féminines dono}

\section{Entre filles, sœurs, mères et épouses}

Les zones dono du Kamma et de Taba-nonu sont, à ma connaissance, les seules régions dogon où les jeunes filles sont rangées dans de véritables classes d'âge, plus de dix ans après leur excision. Les vieilles fixent la composition définitive de cette classe féminine avec pour règle élémentaire de séparer les sœurs germaines ainsi que les jeunes filles partageant le même fiancé (Kervran 1999 : 67-68). Cela montre que le classement des filles obéit en partie à une logique matrimoniale et concerne autant les "sœurs " que les futures épouses à distribuer aux alliés, en leur attribuant à chacune un mari différent.

Au Kamma, la formation des classes d'âge féminines s'articule d'ailleurs à des rites d'initiation préparant les jeunes filles à leur rôle de mère et d'épouse (ibid. : 67-95). Ces rites ont une dimension clanique et territoriale qui anticipe les déplacements matrimoniaux des jeunes filles; ils commencent par un périple féminin au lieu de dispersion de tous les clans du Kamma, puis chez le dépositaire régional du $l \varepsilon b \varepsilon$ (entité incarnant la fertilité de l'ensemble du territoire). Ce long voyage est entrepris par une délégation de six jeunes filles, membres des deux promotions qui précèdent la classe d'âge féminine en cours de formation (et que nous appellerons $\mathrm{C}$, par convention). La plus jeune porte dans le dos, comme un nour-

26. Autrefois, il s'agissait d'un travail d'entraide entièrement gratuit: les filles fumaient les champs de tous les chefs de famille par ordre d'âge décroissant. Aujourd'hui, elles le font uniquement à la demande, en se faisant payer. Cette évolution traduit le glissement de l'association de travail, initialement au service des " aînés", vers un groupement coopératif qui loue ses services. 
risson, une calebasse entière. À leur retour, les filles sont accueillies de nuit par leurs cadettes du groupe $\mathrm{C}$, postées à l'entrée de l'agglomération et protégées de loin par leurs fiancés. Elles demandent aux six jeunes filles de leur "donner le bébé» et elles reçoivent effectivement la calebasse que l'une d'entre elles porte alors jusqu'à l'intérieur du village. Le symbolisme de ce rituel est explicite : la transmission de la calebasse d'une classe d'âge à l'autre annonce les futures maternités des jeunes filles et prépare leur changement de statut.

Par la suite, les cadettes du groupe $\mathrm{C}$ subiront de la part de leurs aînées différentes épreuves et vexations. Elles seconderont également les garçons de la classe d'âge correspondante à la leur, en les aidant par exemple à préparer certaines bières rituelles. Puis, en partant s'installer chez leur mari, elles se disperseront sur l'ensemble du territoire clanique, conçu, au moins autrefois, comme un espace fortement endogame. Les filles d'un nouveau tums se positionnent finalement par rapport à trois groupes différents : leurs aînées (qui incarnent les mères qu'elles vont devenir à l'échelon suivant), leurs " frères » de la classe masculine correspondante (qu'elles vont aider) et leurs fiancés (qui les protègent et qu'elles vont rejoindre ultérieurement). Et c'est en conciliant ces différentes positions - de filles, de sœurs, de mères potentielles et de futures épouses - que les jeunes filles dono forment une classe d'âge.

Selon les âges de la vie, le classement des femmes relève ainsi, alternativement ou simultanément, de la filiation et de l'alliance. Les classes féminines dono se constituent en combinant les deux modèles à un moment transitoire où ses membres sont à la fois des " filles ", résidant encore dans leur village paternel, et des "fiancées", visitant nuitamment leurs futurs époux. Quant aux femmes mariées, dono ou tøne, leur distribution est plus spatiale que temporelle et la répartition de leur charge au sein du village dépend avant tout de leur statut d'épouses «étrangères ", de "sœurs » ou de "nièces utérines ». 
Eric Jolly, Aux fils des âges. Classes d’âge et générations dans cinq régions dogon (Mali). - Cet article réexamine les rapports entre organisation d'âge et formations politiques à travers cinq études de cas, choisis dans différentes régions dogon. Découpées en "échelons initiatiques", les classes d'âge dogon les plus affirmées s'articulent avec un pouvoir politique de type collégial ou "fédéral " autorisant une structure aussi bien lignagère, villageoise que territoriale. En revanche, dès qu'apparait un embryon de chefferie, les regroupements fondés sur l'âge construisent non plus des "promotions d'initiés" mais des "générations" masculines, extérieures à la sphère politique. Dans les deux cas, ce classement reproduit - et redouble - le modèle de la filiation agnatique propre à cette société patrilinéaire et patrilocale en l'étendant, audelà de la parenté, aux unités résidentielles. Lorsqu'ils défilent rangés par classes d'âge ou par générations successives, les hommes d'une localité ou d'un territoire mettent clairement en scène leur auto-engendrement le long d'une chaîne temporelle, mais aussi spatiale.
Eric Jolly, With the Passing of the Ages :Age-sets and Generations in Five Dogon Areas (Mali). The relations between age-group organizations and political formations is examined through five case studies in areas inhabited by the Dogon. Age-sets divided into "initiatory grades" are connected with a collective or "federal " type of political power that allows for autonomous lineage, village and territorial structures. In chiefdoms (even incipient ones) however, age-based groups no longer represent classes of initiates but correspond to masculine generations with no involvement in the political sphere. Each of these two cases reproduces the agnatic descent characteristic of this patrilineal, patrilocal society by extending it beyond kinship to residential units. When, during ceremonies, masculine age-sets or generations from a locality or specific area parade, one man behind the other, this performance clearly represents the temporal and spatial chain of male reproduction. 